\title{
Estimation of the lower and upper bounds on the probability of failure using subset simulation and random set theory
}

\author{
Diego A. Alvarez ${ }^{\mathrm{a}, *}$, Felipe Uribe ${ }^{\mathrm{b}}$, Jorge E. Hurtado ${ }^{\mathrm{a}}$ \\ ${ }^{a}$ Departamento de Ingeniería Civil. Universidad Nacional de Colombia, sede Manizales. \\ Carrera 27 \# 64-60, 170004 Manizales, Colombia. \\ ${ }^{b}$ Engineering Risk Analysis Group, Technische Universität München. \\ Arcisstraße 21, 80333 München, Germany.
}

\begin{abstract}
Random set theory is a general framework which comprises uncertainty in the form of probability boxes, possibility distributions, cumulative distribution functions, Dempster-Shafer structures or intervals; in addition, the dependence between the input variables can be expressed using copulas. In this paper, the lower and upper bounds on the probability of failure are calculated by means of random set theory. In order to accelerate the calculation, a well-known and efficient probability-based reliability method known as subset simulation is employed. This method is especially useful for finding small failure probabilities in both low- and highdimensional spaces, disjoint failure domains and nonlinear limit state functions. The proposed methodology represents a drastic reduction of the computational labor implied by plain Monte Carlo simulation for problems defined with a mixture of representations for the input variables, while delivering similar results. Numerical examples illustrate the efficiency of the proposed approach.

This is an electronic reprint of the original article published by Elsevier in: Mechanical Systems and Signal Processing, 2018, Vol. 100, 782-801

WEB: http://www.sciencedirect.com/science/article/pii/S0888327017304041

DOI: https://doi.org/10.1016/j.ymssp. 2017.07.040 (please cite accordingly).

This reprint differs from the original in pagination and typographic details.
\end{abstract}

Keywords: lower and upper probabilities of failure, imprecise probabilities, random set theory, copulas, interval Monte Carlo, subset simulation, isoprobabilistic transformation.

\section{Introduction}

The treatment of uncertainties and the calculation of the probability of limit state violations are of primary concern in modern engineering systems. Different modes of failure can be grouped in the so-called limit state function (LSF) $g: \mathscr{X} \rightarrow \mathbb{R}$, which depends on a set of uncertain system parameters $\boldsymbol{x} \in \mathscr{X} \subseteq \mathbb{R}^{d}$. In the framework of reliability assessment, the failure surface $g(\boldsymbol{x})=0$ splits the $\mathscr{X}$-space in two domains, namely the safe set $S=\{\boldsymbol{x} \in \mathscr{X}: g(\boldsymbol{x})>0\}$ and the failure set $F=\{\boldsymbol{x} \in \mathscr{X}: g(\boldsymbol{x}) \leq 0\}$. The probability measure of $F \subseteq \mathscr{X}$, also known as the probability of failure, is defined as

$$
P_{\mathrm{f}}=\int_{\mathscr{X}} \mathbb{I}_{F}[\boldsymbol{x}] \mathrm{d} F_{\boldsymbol{X}}(\boldsymbol{x})=\int_{\mathscr{X}} \mathbb{I}[g(\boldsymbol{x}) \leq 0] \mathrm{d} F_{\boldsymbol{X}}(\boldsymbol{x})
$$

where, $F_{\boldsymbol{X}}(\boldsymbol{x})$ is the joint cumulative distribution function $(\mathrm{CDF})$ of the input variables and $\mathbb{I}[\cdot]$ stands for the indicator

\footnotetext{
${ }^{*}$ Corresponding author

Email addresses: daalvarez@unal .edu.co (Diego A. Alvarez), felipe.uribe@tum.de (Felipe Uribe), jehurtadog@unal . edu.co (Jorge E. Hurtado)
}

function, which takes the values $\mathbb{I}_{F}[\boldsymbol{x}]=1$ when either $\boldsymbol{x} \in F$ or the condition in square brackets is true, and $\mathbb{I}_{F}[x]=0$ otherwise. When $F_{\boldsymbol{X}}(\boldsymbol{x})$ is sufficiently differentiable, the associated joint probability density function (PDF) $f_{\boldsymbol{X}}(\boldsymbol{x})$ exists, and in this case equation (1) can be expressed also as

$$
P_{\mathrm{f}}=\int_{\mathscr{X}} \mathbb{I}_{F}[\boldsymbol{x}] f_{\boldsymbol{X}}(\boldsymbol{x}) \mathrm{d} \boldsymbol{x}=\int_{\mathscr{X}} \mathbb{I}[g(\boldsymbol{x}) \leq 0] f_{\boldsymbol{X}}(\boldsymbol{x}) \mathrm{d} \boldsymbol{x} .
$$

One of the main drawbacks of applying the probabilistic approach to reliability analysis of structures is that the CDFs of the input variables $\boldsymbol{x} \in \mathscr{X}$ are usually known with imprecision. This is normally due to the lack of sufficient data for fitting the model to each input random variable. Even if the information is plenty, there remains the problem of the high sensitivity of the usually small probabilities of failure to the parameters of the CDFs (see e.g. Elishakoff (1999); Oberguggenberger and Fellin (2005)). In addition, the information about the input variables, is not always given as joint CDFs, but they can be expressed as well, in terms of probability boxes, possibility distributions, Dempster-Shafer structures, intervals, among other representations of uncertainty. All those reasons complicate the application of the efficient methods that exist in 
the realm of probability theory for the estimation of the reliability of structural and mechanical systems.

These difficulties have fostered the research on alternative methods (coined under the term of imprecise probabilities) for incorporating uncertainty in the analysis of engineering systems, such as possibility theory, info-gap theory, convex models, interval analysis, ellipsoid modeling, credal sets, DempsterShafer evidence theory, random sets, random fuzzy sets, fuzzy random sets, probability boxes, sets with parametrized probability measures, among other methods. The reader is referred to the state-of-the-art review Beer et al. (2013) and references within for further discussion and additional information.

In this context, random set theory appears as a unifying framework which comprises several types of uncertainty representations, either aleatory or epistemic. This approach allows us to estimate the lower and upper bounds on the probability of events, and thus, it can be used to bound the probability of failure. Within the random set approach to structural reliability, research has been done for introducing the epistemic uncertainty in the probabilistic models. In this direction, Tonon (2004) used random set theory to calculate the reliability bounds for the challenge problem proposed by Oberkampf et al. (2004). Du (2008) developed a methodology, termed the unified uncertainty analysis method for reliability assessment of structural and mechanical systems. The approach uses a double loop optimization process which contains probabilistic and interval analysis, and employs the first-order reliability method (FORM) for the solution of the reliability problem. Similarly to the sampling methods developed in Alvarez (2006, 2009a), Zhang and co-workers proposed the so-called interval Monte Carlo simulation method (Zhang et al., 2010, 2017); also they developed an interval importance sampling method (Zhang, 2012) and an interval quasiMonte Carlo method (Zhang et al., 2013). Xiao et al. (2014) proposed an efficient saddle-point approximation to speed up the results of the interval Monte Carlo simulation method. Alvarez and Hurtado (2014) proposed a method based on the reliability plot to estimate in a parsimonious way the lower and upper probabilities of failure. Recently, Yang et al. (2016) used a surrogate kriging model to accelerate the computations of the failure probability bounds.

As it will be seen in the paper, the estimation of the lower and upper probabilities of failure can be postulated as two standard reliability assessment problems, and consequently, any method for the estimation of the probability of failure that only uses probabilistic information can be applied . In particular, we will illustrate this methodology using one of the most popular and efficient methods, namely, subset simulation (Au and Beck, 2001, 2003). During the last decade, subset simulation has established itself as one of the leading algorithms for the estimation of failure probabilities. Therefore, the engineering research community has focused on the enhancement and generalization of the method; some of the most recent contributions include: Bayesian post-processor for subset simulation (Zuev et al., 2012), combination of subset simulation with machine learning-based surrogate models
(Bourinet et al., 2013; Papadopoulos et al., 2013), and the subset simulation enhancements proposed by Papaioannou et al. (2015) and Au and Patelli (2016). Perhaps one of the most significant developments was proposed by Walter (2015), who applied the concept of moving particles; the approach presents two main results in the context of subset simulation (referred to as multilevel splitting): first, the number of samples required to populate $F$ is considerably reduced, and second, the adaptive selection of the intermediate levels is no longer required since by construction the nested subsets do not exist anymore.

In this contribution, we will use subset simulation in conjunction with random set theory in order to estimate the upper and lower bounds on the probability of failure when the input variables are defined in terms of probability boxes, possibility distributions, CDFs, Dempster-Shafer structures, or intervals. In fact, the proposed approach is so general that any method for assessing the probability of failure can be used as well.

The plan of this work is as follows. The document begins with a succinct introduction to copulas and random sets in Sections 2 and 3, respectively. Then in Section 4, we introduce the mathematical formulation for the estimation of the probability of failure and its relationship with random set theory. Specifically, we will see that the calculation of the lower and upper probabilities of failure will correspond to the evaluation of two integrals that compute the probability of failure for two different LSFs. Section 5 will introduce the Monte Carlo simulation method for the estimation of the probability of failure, and Section 6 will introduce the subset simulation algorithm. The proposed methodology will be illustrated in Section 7; there, we explain how to use subset simulation to estimate the lower and upper probabilities of failure provided by random set theory after applying a suitable isoprobabilistic transformation. Section 8 demonstrates the advantages of the proposed approach with three numerical examples. The paper ends with the discussion of results, conclusions, some open problems and the corresponding acknowledgements.

\section{An introduction to copulas}

\subsection{Overview}

This concise review of some important concepts about copulas follows the exposition presented in Nelsen (2007). A copula $C$ is a $d$-dimensional $\mathrm{CDF}, C:[0,1]^{d} \rightarrow[0,1]$, whose univariate marginal $C D F s$ are uniform on the interval $[0,1]$. The main attribute of a copula is to link a given joint CDF with its marginals, carrying all the dependence information of the random variables.

According to the Sklar's theorem (Sklar, 1959), a joint $\mathrm{CDF} F_{\boldsymbol{X}}(\boldsymbol{x})=P\left[X_{1} \leq x_{1}, X_{2} \leq x_{2}, \ldots, X_{d} \leq x_{d}\right]$ with marginal CDFs $F_{X_{k}}\left(x_{k}\right)=P\left[X_{k} \leq x_{k}\right]$ for $k=$ $1, \ldots, d$, can be expressed as $F_{X_{1}, X_{2}, \ldots, X_{d}}\left(x_{1}, x_{2}, \ldots, x_{d}\right)=$ $C\left(F_{X_{1}}\left(x_{1}\right), F_{X_{2}}\left(x_{2}\right), \ldots, F_{X_{d}}\left(x_{d}\right)\right)$. In other words, any joint $\mathrm{CDF}$ can be represented as a function of its univariate marginal 
CDFs and a copula $C$ that contains all information of the dependence structure between the components of the random vector $\boldsymbol{X}=\left[X_{1}, X_{2}, \ldots, X_{d}\right]$.

\section{2. $V_{C}$-volume and Lebesgue-Stieltjes measure $\mu_{C}$}

Let us define a $d$-dimensional box or simply $d$-box $[\boldsymbol{a}, \boldsymbol{b}]$, as the Cartesian product $[\boldsymbol{a}, \boldsymbol{b}]:=\times_{k=1}^{d}\left[a_{k}, b_{k}\right]:=\left[a_{1}, b_{1}\right] \times\left[a_{2}, b_{2}\right] \times$ $\cdots \times\left[a_{d}, b_{d}\right]$, with $a_{k} \leq b_{k}$, for all $k=1,2, \ldots, d$; here $\left[a_{k}, b_{k}\right]_{k=1}^{d}$ represents a set of intervals on the real line.

The $V_{C}$ volume of a $d$-box $[\boldsymbol{a}, \boldsymbol{b}]$ is the $d$-th order difference of the copula $C$ on $[\boldsymbol{a}, \boldsymbol{b}]$, that is,

$$
V_{C}([\boldsymbol{a}, \boldsymbol{b}])=\sum_{i_{1}=1}^{2} \sum_{i_{2}=1}^{2} \cdots \sum_{i_{d}=1}^{2}(-1)^{i_{1}+i_{2}+\cdots+i_{d}} C\left(x_{1 i_{1}}, x_{2 i_{2}}, \ldots, x_{d i_{d}}\right)
$$

where, $\boldsymbol{a}=\left[a_{1}, a_{2}, \ldots, a_{d}\right], \boldsymbol{b}=\left[b_{1}, b_{2}, \ldots, b_{d}\right]$, and $x_{k 1}=$ $a_{k}, x_{k 2}=b_{k}$, for all $k=1,2, \ldots, d$. The $V_{C}$-volume can be understood as the volume associated to the $d$-box $[\boldsymbol{a}, \boldsymbol{b}]$ under the PDF corresponding to the copula $C$ (in the case it exists); in consequence, $V_{C}([\boldsymbol{a}, \boldsymbol{b}]) \geq 0$ for each $[\boldsymbol{a}, \boldsymbol{b}] \subseteq[0,1]^{d}$.

The generalization of the $V_{C}$-volume to sets in $[0,1]^{d}$ that are not $d$-boxes is given by the Lebesgue-Stieltjes measure associated to the copula $C$, denoted $\mu_{C}$. In this case, $\mu_{C}(F)$ is the probability measure of the region $F \in[0,1]^{d}$ under the copula $C$ (see Alvarez (2009a) for further details).

\subsection{Rosenblatt transformation for copulas}

Rosenblatt (1952) proposed a method that transforms a set of dependent random variables $\boldsymbol{\alpha}$ into a set of independent ones $\boldsymbol{\alpha}^{\prime}$. In other words, given the joint CDF of a (possibly dependent) set of random variables, this transform is a one-to-one mapping from that set of variables $\boldsymbol{\alpha}$ onto a set of independently and identically distributed (i.i.d.) random variables $\boldsymbol{\alpha}^{\prime}$.

For copulas, this bijective transform is expressed as follows: for every random vector $\boldsymbol{\alpha}=\left[\alpha_{1}, \ldots, \alpha_{d}\right]$ distributed according to the copula $C:[0,1]^{d} \rightarrow[0,1]$, the Rosenblatt transform $\boldsymbol{\alpha}^{\prime}=T_{\mathrm{Ros}}(\boldsymbol{\alpha})=\left[\alpha_{1}^{\prime}, \ldots, \alpha_{d}^{\prime}\right]$ is given as: $\alpha_{1}^{\prime}=\alpha_{1}$, $\alpha_{2}^{\prime}=C\left(\alpha_{2} \mid \alpha_{1}\right), \ldots, \alpha_{d}^{\prime}=C\left(\alpha_{d} \mid \alpha_{1}, \alpha_{2}, \ldots, \alpha_{d-1}\right)$. In this case, the resulting random vector $\alpha^{\prime}$ takes values in $[0,1]^{d}$ and is uniformly distributed according to the independence copula (also known as product copula), $C\left(\boldsymbol{\alpha}^{\prime}\right)=\prod\left(\boldsymbol{\alpha}^{\prime}\right)=\prod_{k=1}^{d} \alpha_{k}^{\prime}$. Furthermore, the conditional CDFs $C\left(\alpha_{k} \mid \alpha_{1}, \ldots, \alpha_{k-1}\right)$ can be shown to be given by (Schmitz, 2003, p.20),

$$
C\left(\alpha_{k} \mid \alpha_{1}, \ldots, \alpha_{k-1}\right)=\frac{\frac{\partial^{k-1}}{\partial \alpha_{1} \cdots \partial \alpha_{k-1}} C\left(\alpha_{1}, \ldots, \alpha_{k}, 1, \ldots, 1\right)}{\frac{\partial^{k-1}}{\partial \alpha_{1} \cdots \partial \alpha_{k-1}} C\left(\alpha_{1}, \ldots, \alpha_{k-1}, 1, \ldots, 1\right)}
$$

for $k=2, \ldots, d$; this holds under the assumption that $C$ is sufficiently differentiable. By construction, the arguments of the copula in the numerator are filled with ones for the indexes $k+1, \ldots, d$, and the same applies in the denominator for the indexes $k, \ldots, d$. The last equation is often cumbersome to apply in high dimensions, inasmuch as the numerical evaluation of the derivatives might be computationally demanding and is prone to round-off errors.

The inverse of the Rosenblatt transformation $T_{\mathrm{Ros}}^{-1}$ provides the most general sampling method for copulas, which is known as the conditional distribution method (see e.g. Embrechts et al. (2003)). By drawing a set of i.i.d. samples $\alpha_{k}^{\prime}$ for $k=1,2, \ldots, d$ uniformly distributed in $[0,1]$ and using the recursive equations

$$
\begin{aligned}
& \alpha_{1}=\alpha_{1}^{\prime} \\
& \alpha_{k}=C^{-1}\left(\alpha_{k}^{\prime} \mid \alpha_{1}, \alpha_{2}, \ldots, \alpha_{k-1}\right) \text { for } k=2, \ldots, d,
\end{aligned}
$$

a vector $\boldsymbol{\alpha}=T_{\mathrm{Ros}}^{-1}\left(\boldsymbol{\alpha}^{\prime}\right)$ distributed according to the copula $C$ is obtained. Since the computation of the conditional inverses $C^{-1}$ is difficult to handle analytically, the solution of equation (2) can be obtained numerically by solving the nonlinear equation $C\left(\alpha_{k} \mid \alpha_{1}, \ldots, \alpha_{k-1}\right)-\alpha_{k}^{\prime}=0$ for $\alpha_{k} \in[0,1]$.

\section{Random sets: basic concepts}

In the next, a brief review on some ideas on random sets (RS) is presented.

\subsection{Definition of a random set}

Let us consider a universal set $\mathscr{X} \neq \emptyset$ and its power set $\mathscr{P}(\mathscr{X})$, a probability space $\left(\Omega, \sigma_{\Omega}, P_{\Omega}\right)$, and a measurable space $\left(\mathscr{F}, \sigma_{\mathscr{F}}\right)$, where $\mathscr{F} \subseteq \mathscr{P}(\mathscr{X})$. A random set $\Gamma$ is a $\left(\sigma_{\Omega}-\sigma_{\mathscr{F}}\right)$-measurable mapping $\Gamma: \Omega \rightarrow \mathscr{F}, \alpha \mapsto \Gamma(\alpha)$ (Nguyen, 2006; Couso et al., 2014). In other words, a random set is analogous to a random variable whose realization is a set in $\mathscr{F}$, not a number. Let us call each of those sets $\Gamma(\alpha) \in \mathscr{F}$ a focal element and $\mathscr{F}$ a focal set.

Similarly to the definition of a random variable, a random set can be used to define a probability measure on $(\mathscr{F}, \sigma \mathscr{F})$ given by $P_{\Gamma}:=P_{\Omega} \circ \Gamma^{-1}$. That is, an event $\mathcal{R} \in \sigma_{\mathscr{F}}$ has probability $P_{\Gamma}(\mathcal{R})=P_{\Omega}(\{\alpha \in \Omega: \Gamma(\alpha) \in \mathcal{R}\})$. Observe that when all focal elements of $\mathscr{F}$ are singletons, $\Gamma$ becomes a random variable $X$. Hence, $\Gamma(\alpha)=X(\alpha)$ and the probability of occurrence of an event $F$ is $P_{X}(F):=\left(P_{\Omega} \circ X^{-1}\right)(F)=P_{\Omega}\{\alpha \in \Omega: X(\alpha) \in F\}$ for every $F \in \sigma_{\Omega}$.

When applying RS theory, it is not possible to compute exactly $P_{X}(F)$ but only its upper and lower probability bounds, since the exact CDF of the input random variables is unknown. Dempster (1967) defined those lower and upper probabilities by,

$$
\begin{aligned}
& \underline{P_{\Gamma}}(F):=P_{\Omega}(\{\alpha \in \Omega: \Gamma(\alpha) \subseteq F, \Gamma(\alpha) \neq \emptyset\}) \\
& \overline{P_{\Gamma}}(F):=P_{\Omega}(\{\alpha \in \Omega: \Gamma(\alpha) \cap F \neq \emptyset\})
\end{aligned}
$$

where, $\underline{P_{\Gamma}}(F) \leq P_{X}(F) \leq \overline{P_{\Gamma}}(F)$. 


\subsection{Relationship between random sets and other representa- tions of uncertainty}

The definition of a random set in Section 3.1 is very general. Alvarez $(2006,2007)$ proved that particularizing the probability space as,

$-\Omega:=(0,1]^{d}$,

- $\sigma_{\Omega}:=\mathscr{B}(\Omega)$, where $\mathscr{B}(\Omega)$ is the Borel $\sigma$-algebra on $\Omega$,

- $P_{\Omega}:=\mu_{C}$ for some copula $C$ that contains the dependence information within the joint random set, and

- using $d$-boxes as elements of $\mathscr{F}$,

is enough to model several representations of uncertainty, such as, intervals, possibility distributions, CDFs, probability boxes, Dempster-Shafer structures, or their joint combinations.

In the following, we will describe the relationship between RSs and the aforementioned representations of uncertainty. Without loss of generality, the concepts are introduced for onedimensional random sets, that is, $\Omega:=(0,1], \sigma_{\Omega}:=\mathscr{B}(\Omega)$, and $P_{\Omega}$ is a Lebesgue measure on $\Omega$ (the probability measure corresponding to the CDF of a uniformly distributed random variable $\tilde{\alpha}$ on $(0,1])$. Joint (multidimensional) random sets will be analyzed from Section 3.3 on.

\section{RSs and intervals}

An interval $I=[a, b]$ models a range of values between a lower value $a$ and an upper value $b$. They do not involve any knowledge of the CDFs of the values within $a$ and $b$, in fact, all CDFs in $I$ are possible. In consequence, intervals should not be confused with a uniform $\mathrm{CDF}$ in $[a, b]$, inasmuch as that would imply commuting imprecision with randomness. An interval $I$ can be represented by the random set $\Gamma(\alpha):=I$, that is, all $\alpha \in \Omega$ draw the same interval $I$.

\section{RSs and possibility distributions}

A possibility distribution is expressed as a normalized fuzzy set, with membership function $A: X \rightarrow(0,1], X \subseteq \mathbb{R}$ (Dubois and Prade, 1988). It can be represented as the random set $\Gamma: \Omega \rightarrow \mathscr{F}$, whose focal set $\mathscr{F}$ is the system of all $\alpha$-cuts of the membership function $A$, that is, $\Gamma(\alpha):=\{x \in X: A(x) \geq \alpha\}$ for $\alpha \in(0,1]$.

\section{RSs and cumulative distribution functions}

Consider a random variable on $X: \Omega \rightarrow \mathbb{R}$; the probability law of the random variable can be expressed using its CDF $F_{X}$. The CDF of the random variable $X$ can be represented by the random set $\Gamma(\alpha):=F_{X}^{(-1)}(\alpha)$ for $\alpha \in \Omega$, where, $F_{X}^{(-1)}(\alpha):=$ $\inf \left\{x: F_{X}(x) \geq \alpha\right\}$ denotes the quasi-inverse of $F_{X}$.

\section{RSs and probability boxes}

A probability box or simply $p$-box is the set of CDFs $\left\{F_{X}\right.$ : $\left.F_{X}(x) \leq F_{X}(x) \leq \overline{F_{X}}(x), x \in \mathbb{R}\right\}$ which conjointly represents the uncertainty about the true CDF associated to a random variable $X$ (Ferson et al., 2003). According to Crespo et al. (2013), probability boxes can be classified into two disjoint groups: distributional and distribution-free, which are discussed next.

Distributional p-boxes, also known as parametric, result when there is uncertainty in the representation of the parameters of the parental CDF (see below). Let us consider the CDF $F_{X}(x ; \boldsymbol{\theta})$ associated to a random variable $X$ whose vector of parameters is $\boldsymbol{\theta}=\left[\theta_{1}, \ldots, \theta_{m}\right]$. When the parameters $\boldsymbol{\theta}$ are imprecisely specified as intervals, such that $\theta_{i} \in I_{i}$ for $i=1, \ldots, m$, the set of all CDFs whose parameters comply with those intervals form the distributional p-box $\left\{F_{X}(x ; \boldsymbol{\theta}): \boldsymbol{\theta} \in I_{1} \times \cdots \times I_{m}\right\}$; here $F_{X}$ represents the socalled parental CDF. For instance, consider a Gaussian distributed random variable with mean in the interval $[0,2]$ and variance in $[1,3]$. Only those Gaussian CDFs whose mean and variance lie in those intervals will belong to the p-box; in this case the parental CDF of the p-box is Gaussian distributed and no other family type of CDFs within the p-box will be allowed.

Distribution-free p-boxes, also known as non-parametric, appear when the parental family of the CDF that specifies the p-box is unknown. In this case, only the CDF bounds $F_{X}$ and $\overline{F_{X}}$ are clearly and explicitly stated, and no assumptions about the parental CDF, or shape of the uncertain CDFs are made. Alvarez (2006) showed that this type of p-box can be specified as the random set $\Gamma(\alpha):=\left[{\overline{F_{X}}}^{-1}(\alpha), F_{X}^{-1}(\alpha)\right]$ for $\alpha \in \Omega$. Distribution-free pboxes do not retain information about the internal structure within the CDF bounds $F_{X}$ and $\overline{F_{X}}$, and in consequence, they do not elucidate which distributions within the $\mathrm{p}$-box are the most likely.

Distributional probability boxes are a useful tool to model CDFs whose parameters are known up to intervals. However, when used together with random set theory, they must be converted to distribution-free probability boxes, with an associated loss of information. This is because the inherent inability of random set theory to specify the parental CDF related to the probability box. A distributional p-box can be approximated by a distribution-free p-box using for instance the method described in Alvarez et al. (2017). In the same reference, it is also devised a method to estimate the bounds of the probability of failure dealing directly with distributional p-boxes; as a result, tighter bounds on the probability of failure are provided since the additional information included with the approximation of distributional p-boxes as distribution-free ones is excluded in the calculation.

Finally, Dempster-Shafer structures are also closely related to random sets. The reader is referred to $\operatorname{Alvarez}(2006,2007)$ for further information. 


\subsection{Sampling from a random set}

Let us consider again the probability space $\left(\Omega, \sigma_{\Omega}, P_{\Omega}\right)$ with $\Omega:=(0,1], \sigma_{\Omega}:=\mathscr{B}(\Omega)$ and $P_{\Omega}$ a Lebesgue measure on $(0,1]$. A sample of a one-dimensional random set is simply obtained by generating an $\alpha$ from the uniform distribution in $(0,1]$ and then computing the corresponding focal element $\Gamma(\alpha)$.

An extension to higher dimensions, $\Omega:=(0,1]^{d}, \sigma_{\Omega}:=\mathscr{B}(\Omega)$ and $P_{\Omega}:=\mu_{C}$, can be carried out by sampling an $\alpha$ from the copula $C$ and then combining the associated focal elements. These joint focal elements are given by the Cartesian product $\mathrm{X}_{k=1}^{d} \Gamma_{k}\left(\alpha_{k}\right)$, where $\Gamma_{k}\left(\alpha_{k}\right)$ are the sampled focal elements from every basic variable. Note that some of these $\Gamma_{k}\left(\alpha_{k}\right)$ are intervals, some other points. Thus, considering that every sample of a basic variable can be portrayed either by $\Gamma_{k}\left(\alpha_{k}\right)$ or by the corresponding $\alpha_{k}$, the joint focal element can be represented either by the $d$-box $\chi_{k=1}^{d} \Gamma_{k}\left(\alpha_{k}\right) \subseteq \mathscr{X}$ or by the point $\boldsymbol{\alpha}=\left[\alpha_{1}, \alpha_{2}, \ldots, \alpha_{d}\right] \in(0,1]^{d}=\Omega$, as illustrated in Figure 1a and Figure 1b, respectively.

\subsection{Lower and upper probabilities of the failure event $F$}

Under the particularizations of Section 3.2, the sample space $\Omega$ contains the regions (Alvarez, 2006),

$$
\begin{aligned}
& F_{L P}:=\{\alpha \in \Omega: \Gamma(\alpha) \subseteq F, \Gamma(\alpha) \neq \emptyset\} \\
& F_{U P}:=\{\alpha \in \Omega: \Gamma(\alpha) \cap F \neq \emptyset\}
\end{aligned}
$$

which are correspondingly formed by all those points in $\Omega$ whose respective focal elements are completely contained in the set $F$ or have at least one point in common with $F$ (see Figure 1a). These sets are called respectively the lower and upper inverses of $F$ under the random set $\Gamma$. In particular observe that $F_{L P} \subseteq F_{U P}$.

In this case, the lower (3a) and upper (3b) probability bounds of $F$ can be calculated by the Lebesgue-Stieltjes integrals (Alvarez, 2006, 2009a)

$$
\begin{aligned}
& \underline{P_{\Gamma}}(F)=\int_{\Omega} \mathbb{I}_{F_{L P}}[\alpha] \mathrm{d} C(\boldsymbol{\alpha})=\mu_{C}\left(F_{L P}\right) \\
& \overline{P_{\Gamma}}(F)=\int_{\Omega} \mathbb{I}_{F_{U P}}[\boldsymbol{\alpha}] \mathrm{d} C(\boldsymbol{\alpha})=\mu_{C}\left(F_{U P}\right),
\end{aligned}
$$

provided that $F_{L P}$ and $F_{U P}$ are $\mu_{C}$-measurable sets. This measurability is guaranteed by the fundamental measurability theorem Molchanov (2005).

\subsection{Finding the image of a focal element through a function}

In the context of structural reliability, it is required to estimate the image of a focal element $\Gamma(\boldsymbol{\alpha})$ through the LSF $g$, that is, $g(\Gamma(\alpha))$. This mapped focal element is obtained through the application of the so-called extension principle (Dubois and Prade, 1991), so that

$$
g\left(\Gamma\left(\boldsymbol{\alpha}_{i}\right)\right)=\left[\underline{g}\left(\boldsymbol{\alpha}_{i}\right), \bar{g}\left(\boldsymbol{\alpha}_{i}\right)\right]
$$

where, $\underline{g}$ and $\bar{g}$ are the LSFs defined in the $\Omega$-space (see Figure $1 b)$ and are given by,

$$
\underline{g}\left(\boldsymbol{\alpha}_{i}\right):=\min _{\boldsymbol{x} \in \Gamma\left(\boldsymbol{\alpha}_{i}\right)} g(\boldsymbol{x}) \quad \text { and } \quad \bar{g}\left(\boldsymbol{\alpha}_{i}\right):=\max _{\boldsymbol{x} \in \Gamma\left(\boldsymbol{\alpha}_{i}\right)} g(\boldsymbol{x}) ;
$$

these are optimization problems that can be solved using any nonlinear constrained global optimization algorithm. However, since this procedure is computationally expensive and suffers the curse of dimensionality, alternative approaches, such as the sampling method, the vertex method, the function approximation method, interval arithmetic or the approach proposed in Hurtado et al. (2012) are usually employed in order to reduce the computational load (see e.g. Alvarez (2007)).

\section{Random sets and the bounding of the probability of fail- ure}

In the context of random set theory, we cannot compute an exact value of the probability of failure $P_{\mathrm{f}}$, inasmuch as the exact distribution of the input variables is not known beforehand. However, as shown in Alvarez (2006, 2009a), it is possible to estimate the lower and upper probabilities of failure using equations (5a) and (5b) as

$$
\underline{P_{\mathrm{f}}}=\underline{P_{\Gamma}}(F), \quad \overline{P_{\mathrm{f}}}=\overline{P_{\Gamma}}(F)
$$

respectively. Moreover, from equations (4) and (6), it can be seen that the following relations apply,

$$
\begin{aligned}
& \mathbb{I}[\Gamma(\boldsymbol{\alpha}) \subseteq F]=\mathbb{I}_{F_{L P}}[\boldsymbol{\alpha}]=\mathbb{I}[\bar{g}(\boldsymbol{\alpha}) \leq 0] \\
& \mathbb{I}[\Gamma(\boldsymbol{\alpha}) \cap F \neq \emptyset]=\mathbb{I}_{F_{U P}}[\boldsymbol{\alpha}]=\mathbb{I}[\underline{g}(\boldsymbol{\alpha}) \leq 0]
\end{aligned}
$$

and therefore, the bounds on the probability of failure in equations (7) can be written as Alvarez and Hurtado (2014):

$$
\begin{aligned}
& \underline{P_{\mathrm{f}}}=\int_{\Omega} \mathbb{I}[\bar{g}(\boldsymbol{\alpha}) \leq 0] \mathrm{d} C(\boldsymbol{\alpha}) \\
& \overline{P_{\mathrm{f}}}=\int_{\Omega} \mathbb{I}[\underline{g}(\boldsymbol{\alpha}) \leq 0] \mathrm{d} C(\boldsymbol{\alpha}) .
\end{aligned}
$$

By comparing equations (1) and (9), we realize that the estimation of $P_{\mathrm{f}}$ and $\overline{P_{\mathrm{f}}}$ reduces to assessing the probability of failure in the $\Omega$-space for two different LSFs, namely $g$ and $\bar{g}$. This is an important fact that allows us to use the already existing methods for the estimation of failure probabilities within random set theory, with minor modifications.

\section{Estimation of $\boldsymbol{P}_{\mathrm{f}}$ and $\overline{\boldsymbol{P}_{\mathrm{f}}}$ using Monte Carlo simulation}

Monte Carlo simulation (MCS) methods are usually employed to evaluate the integral (1). The main idea of the MCS approach is to solve the target problem by directly simulating the numerical model a given number of times, obtaining a sequence of solutions which is used to estimate statistics of the system response. In structural reliability, MCS generates i.i.d. samples 


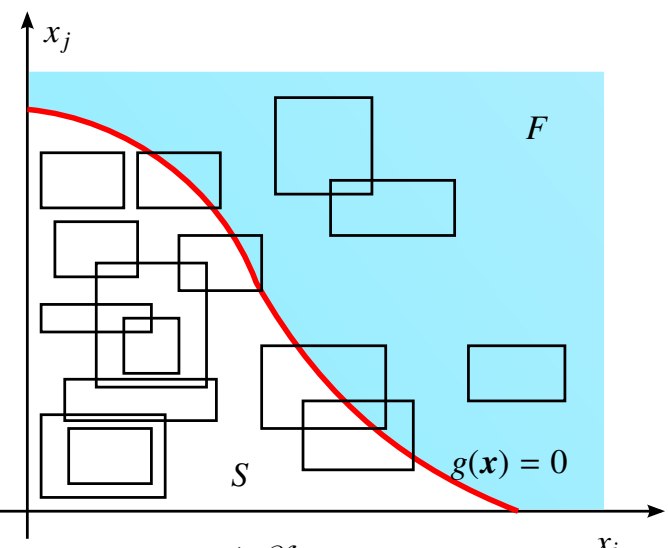

a) $\mathscr{X}$-space

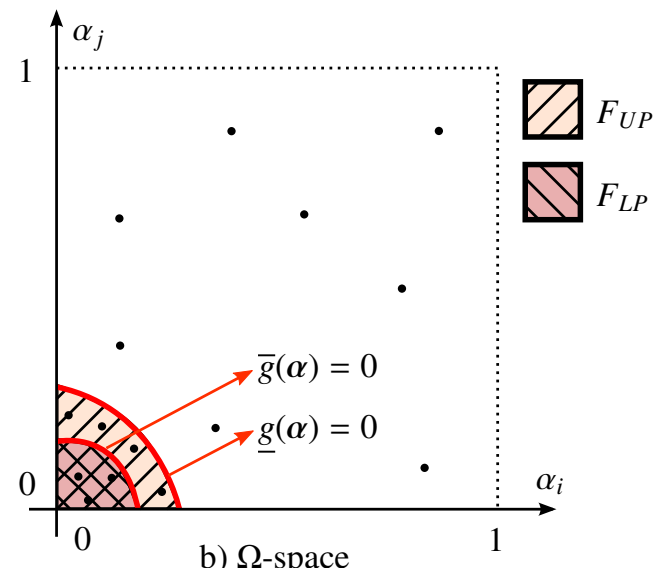

b) $\Omega$-space

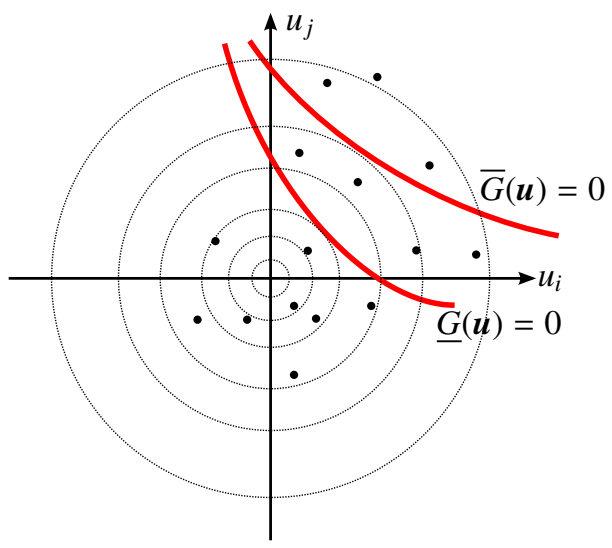

c) $\mathscr{U}$-space

Figure 1: Panel a): the basic variables are defined in the space $\mathscr{X}$; the realizations of these variables by means of random set theory are the focal elements which are depicted as boxes; it is also shown the failure surface $g(\boldsymbol{x})=0$ together with the safe $S$ and failure $F$ domains. Panel b): the copula $C$ is defined in the space $\Omega$; in this space are defined the regions $F_{L P}$ and $F_{U P}$ together with the failure surfaces $g(\boldsymbol{\alpha})=0$ and $\bar{g}(\boldsymbol{\alpha})=0$ (see Eqs. (6)). Notice that $F_{L P} \subseteq F_{U P}$. Panel c): the standard Gaussian space $\mathscr{U}$ together with the failure surfaces $\underline{G}(\boldsymbol{u})=0$ and $\bar{G}(\boldsymbol{u})=0$ (see Eqs. (12)).

from the joint $\mathrm{CDF}$ of the input variables $F_{X}(\boldsymbol{x})$, to obtain a large set of LSF values with the purpose of estimating the failure probability as a sample average.

The generation of samples might be carried out using the inverse transform method, as follows: first, $N$ points $\alpha_{i}=$ $\left[\alpha_{i}^{(1)}, \ldots, \alpha_{i}^{(k)}, \ldots, \alpha_{i}^{(d)}\right]$ for $i=1,2, \ldots, N$ are simulated from the copula $C$, e.g., using the conditional distribution method explained in Section 2.3 (Nelsen (2007) provides additional methods for sampling from copulas); thereafter, each marginal $\left.\operatorname{CDF}\left\{F_{X_{k}}\right\}\right|_{k=1} ^{d}$ is used to obtain the realization $x_{i}^{(k)}=F_{X_{k}}^{-1}\left(\alpha_{i}^{(k)}\right)$. The point $\boldsymbol{x}_{i}=\left[x_{i}^{(1)}, \ldots, x_{i}^{(k)}, \ldots, x_{i}^{(d)}\right]$ will serve as a sample of the joint $\mathrm{CDF} F_{X}$; finally, the MCS estimate of the probability of failure (1) is computed as

$$
\hat{P}_{\mathrm{f}}=\frac{1}{N} \sum_{i=1}^{N} \mathbb{I}_{F}\left[\boldsymbol{x}_{i}\right]=\frac{1}{N} \sum_{i=1}^{N} \mathbb{I}\left[g\left(\boldsymbol{x}_{i}\right) \leq 0\right] .
$$

When considering RSs, for each $\alpha_{i}$ drawn from the copula $C$, we obtain a corresponding focal element $\Gamma\left(\alpha_{i}\right)$; this is a $d$-box resulting from the Cartesian product of the unidimensional focal elements obtained for each input variable. In consequence, this joint focal element contains all possible realizations of the input variables for a given $\boldsymbol{\alpha}_{i}$. Therefore, in Alvarez (2006, 2007, 2009a), it is shown that integrals (9) can be computed using the unbiased and consistent estimators

$$
\begin{aligned}
& \hat{\hat{P}_{\mathrm{f}}}=\frac{1}{N} \sum_{i=1}^{N} \mathbb{I}_{F_{L P}}\left[\boldsymbol{\alpha}_{i}\right]=\frac{1}{N} \sum_{i=1}^{N} \mathbb{I}\left[\bar{g}\left(\boldsymbol{\alpha}_{i}\right) \leq 0\right] \\
& \hat{P_{\mathrm{f}}}=\frac{1}{N} \sum_{i=1}^{N} \mathbb{I}_{F_{U P}}\left[\boldsymbol{\alpha}_{i}\right]=\frac{1}{N} \sum_{i=1}^{N} \mathbb{I}\left[\underline{g}\left(\boldsymbol{\alpha}_{i}\right) \leq 0\right] .
\end{aligned}
$$

According to (8), $\Gamma(\boldsymbol{\alpha}) \nsubseteq F$ and $\bar{g}(\boldsymbol{\alpha})>0$ are equivalent expressions; also $\Gamma(\boldsymbol{\alpha}) \cap F=\emptyset$ and $g(\boldsymbol{\alpha})>0$ are equivalent. Moreover, since $\Gamma(\boldsymbol{\alpha}) \cap F=\emptyset$ implies that $\Gamma(\boldsymbol{\alpha}) \nsubseteq F$, hence, $g(\boldsymbol{\alpha})>0$ entails $\bar{g}(\boldsymbol{\alpha})>0$. This fact can be used in order to largely reduce the number of evaluations of the function $\bar{g}$. If the same set of samples $\alpha_{i}$ from the copula $C$ is employed in the evaluation 
of equations (10), it is not necessary to evaluate $\bar{g}$ for the samples that satisfy $\mathbb{I}\left[g\left(\boldsymbol{\alpha}_{i}\right) \leq 0\right]=0$ because this automatically indicates $\mathbb{I}\left[\bar{g}\left(\boldsymbol{\alpha}_{i}\right) \leq 0\right]=0$. Therefore, once equation (10b) is computed, only those samples $\boldsymbol{\alpha}_{i}$ which fulfill $\underline{g}\left(\boldsymbol{\alpha}_{i}\right) \leq 0$ are employed in the evaluation of $\bar{g}$ in equation (10a).

Equations (10) are simply the implementation of the so-called interval Monte Carlo method, after Zhang et al. (2010, 2013, 2017). Note that Zhang and co-workers originally formulated their method assuming independence between the input variables, that is, they have assumed a product copula $\prod(\boldsymbol{\alpha})$ and have performed the sampling using simple MCS and deterministic low-discrepancy sequences. In this sense, the method proposed by Zhang and co-workers is a particularization of the one proposed by Alvarez (2006, 2009a) when the focal sets are mapped through the function $g$ using the optimization method, inasmuch as the latter includes dependence between the basic variables and also supports not only p-boxes and CDFs but also possibility distributions, intervals and Dempster-Shafer structures.

As we will show in Section 7, integrals (9) can also be estimated by any advanced Monte Carlo simulation method, e.g., subset simulation, line sampling, importance sampling, adaptive sampling, etc. In particular, we will illustrate this approach using subset simulation.

\section{Review of subset simulation}

\subsection{Description of the method in the $\mathscr{U}$-space}

The subset simulation (SuS) method, proposed by Au and Beck (2001), is a stochastic simulation algorithm for reliability analysis of engineering systems whose failure probabilities strongly depend on the realization of rare events. The main advantage of this approach lies in its ability to transform a rare event simulation problem into a sequence of problems involving more frequent events. This process is carried out by expressing a very small probability of failure as the product of larger conditional probabilities in the following way: the standard Gaussian space $\mathscr{U}$ is divided into a decreasing sequence of nested subsets (referred to as intermediate failure domains) starting from the whole space and narrowing down to the target failure set, i.e., $\mathscr{U}=\mathcal{F}_{0} \supset \mathcal{F}_{1} \supset \cdots \supset \mathcal{F}_{m}=\mathcal{F}$, such that $\mathcal{F}=\bigcap_{j=0}^{m} \mathcal{F}_{j}$. Here, the failure set is defined by the LSF $G: \mathscr{U} \rightarrow \mathbb{R}$ as $\mathcal{F}=\{\boldsymbol{u} \in \mathscr{U}: G(\boldsymbol{u}) \leq 0\}$.

Consequently, by the general product rule of probability, the probability of failure can be expressed as (Au and Beck, 2001),

$$
\begin{aligned}
P_{\mathrm{f}}=P\left(\cap_{j=0}^{m} \mathcal{F}_{j}\right) & =P\left(\mathcal{F}_{m} \mid \cap_{j=0}^{m-1} \mathcal{F}_{j}\right) P\left(\cap_{j=0}^{m-1} \mathcal{F}_{j}\right) \\
& =P\left(\mathcal{F}_{m} \mid \mathcal{F}_{m-1}\right) P\left(\cap_{j=0}^{m-1} \mathcal{F}_{j}\right) \\
& =\cdots=\prod_{j=1}^{m} P\left(\mathcal{F}_{j} \mid \mathcal{F}_{j-1}\right) ;
\end{aligned}
$$

here, $P\left(\mathcal{F}_{j} \mid \mathcal{F}_{j-1}\right)$ represents the conditional probability at the $(j-1)$-th level. Each intermediate failure domain is defined as the set $\mathcal{F}_{j}=\left\{\boldsymbol{u} \in \mathscr{U}: G(\boldsymbol{u}) \leq b_{j}\right\}$, where $b_{1}>\cdots>b_{j}>$ $\cdots>b_{m}=0$ forms a decreasing sequence of threshold levels. The set of samples that belongs to the level $\mathcal{F}_{j}$ is denoted as $\boldsymbol{u}_{j}$; more precisely, the notation $u_{j, i}^{(k)}$ indicates the $k$-th component of the $i$-th sample that belongs to the level $j$. The conditional probabilities can be estimated by MCS as,

$$
P\left(\mathcal{F}_{j} \mid \mathcal{F}_{j-1}\right) \approx \hat{P}_{j}=\frac{1}{N} \sum_{i=1}^{N} \mathbb{I}_{\mathcal{F}_{j}}\left[\boldsymbol{u}_{j-1, i}\right],
$$

where, $\left.\left\{\boldsymbol{u}_{j-1, i}\right\}\right|_{i=1} ^{N}$ are samples distributed according to the conditional standard Gaussian PDFs $\phi_{\boldsymbol{U}}\left(\cdot \mid \mathcal{F}_{j-1}\right)$. Observe that the initial samples $\left.\left\{\boldsymbol{u}_{0, i}\right\}\right|_{i=1} ^{N}$ are i.i.d. distributed according to the joint standard Gaussian $\operatorname{PDF} \phi_{U}(\cdot)=\phi_{U}\left(\cdot \mid \mathcal{F}_{0}\right)$. However, at higher intermediate levels, the calculation of $P\left(\mathcal{F}_{j} \mid \mathcal{F}_{j-1}\right)$ for $j=2, \ldots, m$ is more complicated since it is necessary to draw i.i.d. samples $\left.\left\{\boldsymbol{u}_{j-1, i}\right\}\right\}_{i=1}^{N}$ from the conditional densities $\phi_{U}\left(\cdot \mid \mathcal{F}_{j-1}\right)$. SuS handles this task by generating samples with especially designed Markov chains that sequentially populate the target failure domain $\mathcal{F}$. This requires the application of a Markov chain Monte Carlo (MCMC) algorithm (this will be discussed in Section 6.2).

In practical applications, it is not possible to make an optimal a priori selection of the intermediate threshold values. Therefore, they are adaptively selected in such a way that the conditional failure probability of each level corresponds to a common fixed value $P\left(\mathcal{F}_{j} \mid \mathcal{F}_{j-1}\right)=p_{0}$. Experience suggests that $p_{0} \in[0.1,0.3]$ yields good efficiency; moreover, for implementation reasons, $p_{0}$ is chosen such that $N_{s}=N p_{0}$ and $N_{c}=1 / p_{0}$ are natural numbers (Zuev et al., 2012). Observe that this adaptive selection implies that each threshold value $b_{j}$ corresponds to the $p_{0}$-percentile of the set of LSF values $G\left(\boldsymbol{u}_{j-1}\right)$.

Provided that a suitable MCMC method is used for sampling the intermediate conditional distributions, the SuS method proceeds as follows: from the initial MCS stage, the first intermediate level $b_{1}$ is selected as the $p_{0}$-percentile of the LSF values $G\left(\boldsymbol{u}_{0}\right)$, by doing so, $N_{s}$ samples from $\boldsymbol{u}_{0}$ that lie in $\mathcal{F}_{1}$ will be distributed according to $\phi_{U}\left(\cdot \mid \mathcal{F}_{1}\right)$. Those samples provide seeds to simulate Markov chains using a MCMC algorithm that implicitly has $\phi_{\boldsymbol{U}}\left(\cdot \mid \mathcal{F}_{1}\right)$ as stationary distribution; this process generates new samples $\left.\left\{\boldsymbol{u}_{1, i}\right\}\right|_{i=1} ^{N}$. In the same way, the level $b_{2}$ is selected as the $p_{0}$-percentile of the LSF values $G\left(\boldsymbol{u}_{1}\right)$ and $N_{s}$ samples from $\boldsymbol{u}_{1}$ that lie in $\mathcal{F}_{2}$ will be distributed according to $\phi_{U}\left(\cdot \mid \mathcal{F}_{2}\right)$. By repeating this process until the failure event $\mathcal{F}$ is reached, the failure probability estimator of the $\mathrm{SuS}$ method is given by,

$$
\hat{P}_{\mathrm{f}}=\prod_{j=1}^{m} \hat{P}_{j}=p_{0}^{m-1} \frac{\sum_{i=1}^{N} \mathbb{I}_{\mathcal{F}_{m}}\left[\boldsymbol{u}_{m-1, i}\right]}{N}
$$

where the second term of the right hand side, corresponds to the intermediate conditional probability at the last level $b_{m}=0$ computed as the ratio between the number of samples that lie in $\mathcal{F}=\mathcal{F}_{m}$ and the total number of samples per level $N$. A general 
procedure for the implementation of the SuS method is shown Algorithm 1.

\subsection{MCMC algorithms for subset simulation}

The objective of MCMC methods is the generation of samples from arbitrary complex distributions. It should be noted that MCMC samples are no longer independent; nevertheless, they can still be used in the computation of the conditional probabilities at the expense of a reduction in efficiency (Zuev et al., 2012).

For subset simulation in the $\mathscr{U}$-space, MCMC algorithms are designed in such a way that the Markov chains have the intermediate conditional densities $\phi_{\boldsymbol{U}}\left(\cdot \mid \mathcal{F}_{j}\right)$ as their stationary distributions. The first step of the simulation takes a sample that belongs to $\mathcal{F}_{j}$ (called seed) and computes a random walk

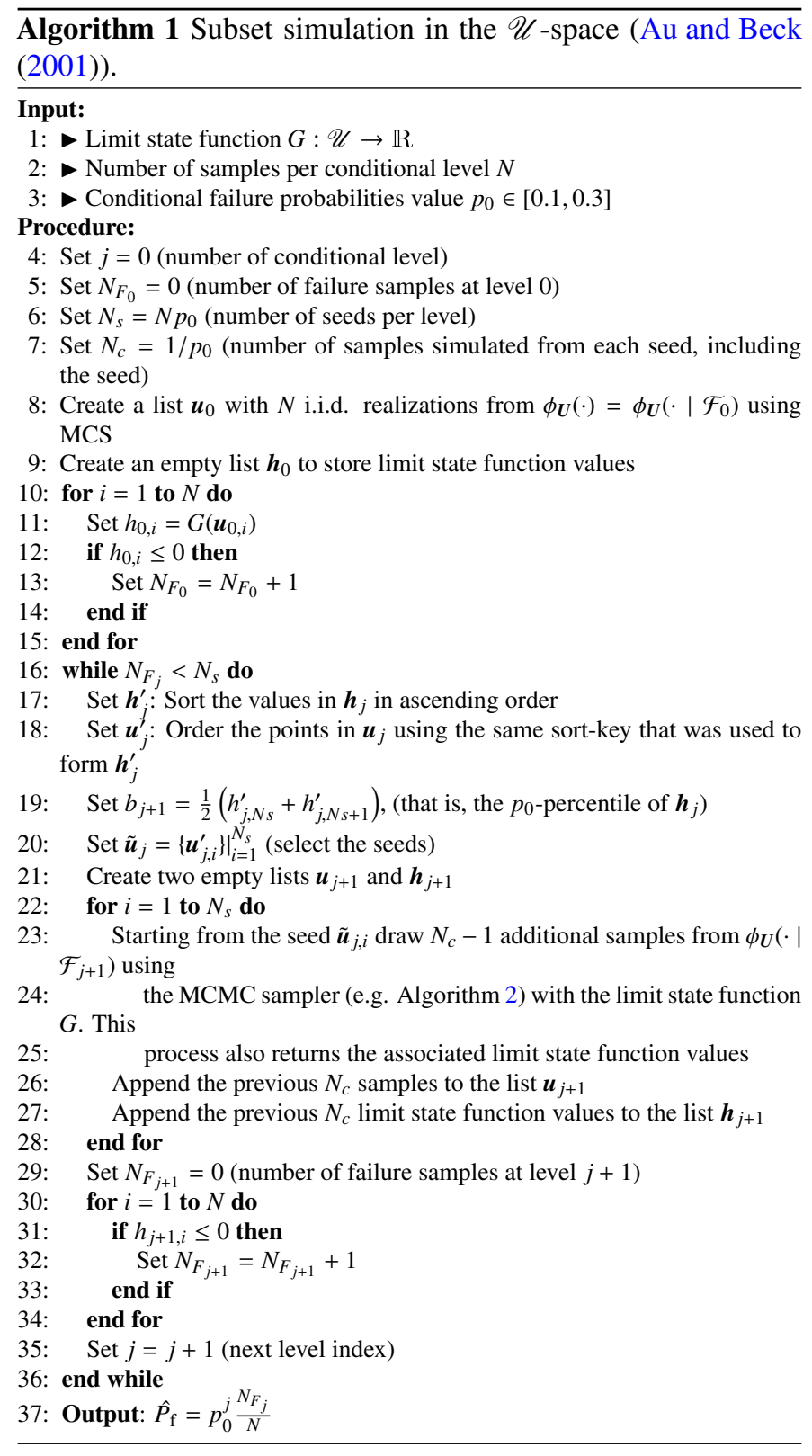

in order to generate a candidate sample also distributed according to $\phi_{\boldsymbol{U}}\left(\cdot \mid \mathcal{F}_{j}\right)$. The second step ensures that the candidate sample lies in the intermediate failure level $\mathcal{F}_{j}$ by performing a LSF evaluation. Since the seeds are always distributed according to the conditional density (due to the adaptive selection of the intermediate levels), the samples generated during the MCMC simulation are also distributed according to this density. Hence, it is realized that all the Markov chains generated in $\mathrm{SuS}$ are in stationary state from the beginning of the simulation, which avoids the implementation of the common MCMC burn-in stages. This property of the MCMC samplers in SuS is referred to as perfect sampling (Au et al., 2012).

Traditional modified Metropolis-Hastings (MMH) algorithms in the context of $\mathrm{SuS}$ are adapted and/or improved versions of the original $\mathrm{MH}$ procedure, since it has been found that the latter has difficulties simulating random variables with many components, thus becoming very inefficient in high dimensional problems (Au and Beck, 2003). MMH methods (also know as component-wise) overcome this deficiency by using a sequence of one-dimensional proposal distributions to generate the candidate state one component at a time, instead of using a high dimensional proposal to obtain the candidate samples; some of these methods include: modified Metropolis algorithm (Au and Beck, 2001), modified Metropolis-Hastings with grouping of samples (Au and Beck, 2003), modified Metropolis-Hasting with delayed rejection (Zuev and Katafygiotis, 2011), adaptive conditional sampling Papaioannou et al. (2015), among others. Recently, by noting that the dimensionality of the problem increases the rejection of candidate states in the MCMC sampler of SuS, Au and Patelli (2016) proposed a so-called limiting algorithm to generate Gaussian candidate samples expressed in terms of an arbitrary increasing number of Gaussian random variables. According to $\mathrm{Au}$ and Patelli, their method tackles the problem of generating conditional samples in the standard Gaussian space from a more general perspective than Papaioannou et al. (2015), but generally both methods coincide.

In the following, we will employ the adaptive conditional sampling method as the MCMC sampler of SuS since it allows us to reduce the sample correlation. This gives a corresponding reduction in the coefficient of variation (c.o.v.) of the probability of failure estimates, with a similar computational cost compared to the original modified Metropolis algorithm (MMA) by Au and Beck (2001). Moreover, compared to other MCMC methods for SuS, the candidate state is always accepted at the first step of the simulation.

Adaptive conditional sampling (ACS) (Papaioannou et al., 2015): in a Metropolis-Hastings algorithm setting, given a seed sampled at level $j$ (that also belongs to the level $j+1$ ), denoted $\tilde{\boldsymbol{u}}_{j, i} \in \mathcal{F}_{j} \cap \mathcal{F}_{j+1}$, for $j=0, \ldots, m-1$ and $i=1, \ldots, N_{s}$, the candidate state $\boldsymbol{u}^{*}$ is drawn from the $\operatorname{PDF} \phi_{\boldsymbol{U}}\left(\cdot \mid \mathcal{F}_{j+1}\right)$. The idea of the conditional sampling (CS) method is to impose that the candidate state and the seed are jointly Gaussian distributed with component-wise correlation coefficient $\rho_{k}$. Therefore, writing out a new random vector with components $\left[\boldsymbol{u}^{*} ; \tilde{\boldsymbol{u}}_{j, i}\right]^{\top}$, we 
are now interested in the conditional distribution $\phi_{\boldsymbol{U}}\left(\boldsymbol{u}^{*} \mid \tilde{\boldsymbol{u}}_{j, i}\right)$. Notice that the mean vectors and covariance matrices of this random vector can be partitioned accordingly in terms of the individual and crossed components. In this case, the vector $\left[\boldsymbol{u}^{*} ; \tilde{\boldsymbol{u}}_{j, i}\right]^{\top}$ is distributed according to

$$
\mathrm{N}\left(\left[\begin{array}{l}
\mathbf{0} \\
\mathbf{0}
\end{array}\right],\left[\begin{array}{cc}
\boldsymbol{I} & \boldsymbol{R} \\
\boldsymbol{R}^{\top} & \boldsymbol{I}
\end{array}\right]\right)
$$

where, $\boldsymbol{I} \in \mathbb{R}^{d \times d}$ is the identity matrix and the cross-covariance matrix $\boldsymbol{R} \in \mathbb{R}^{d \times d}$ is a diagonal matrix with its $k$-th diagonal term equal to the correlation coefficient $\rho_{k}$. Based on this partition, it can be shown (Vanmarcke, 2010, p. 48) that the conditional distribution $\phi_{\boldsymbol{U}}\left(\boldsymbol{u}^{*} \mid \tilde{\boldsymbol{u}}_{j, i}\right)$ is given by the $d$-dimensional Gaussian PDF, $\mathrm{N}\left(\boldsymbol{R} \tilde{\boldsymbol{u}}_{j, i}, \mathbf{I}-\mathbf{R R}^{\top}\right)$. Because of the independence, each $k$-th component of this conditional distribution is given by a one-dimensional Gaussian with mean value $\rho_{k} \tilde{u}_{j, i}^{(k)}$ and variance $1-\rho_{k}^{2}$, for $k=1, \ldots, d$. If not specified, the squared correlation coefficients $\rho_{k}^{2}$ can be estimated from the sample standard deviation $\tilde{\boldsymbol{\sigma}}_{j} \in \mathbb{R}^{d \times 1}$ of the seeds $\tilde{\boldsymbol{u}}_{j}$. Then, each term of the squared correlation parameter is estimated as $\rho_{k}^{2}=1-\sigma_{k}^{2}$, where $\sigma_{k}=\min \left(1, \tilde{\sigma}_{j}^{(k)}\right)$ (here, the min function is required since $\sigma_{k}^{2}$ cannot be larger than the standard deviations of the marginals of the PDF $\phi_{U}$, which are one).

As in most of MCMC samplers, the efficiency of the CS method is controlled by the spread of the proposal distribution, which in this case depends on the choice of the correlation parameters $\rho_{k}$. A small $\rho_{k}$ decreases the acceptance rate of the candidate samples, while a large $\rho_{k}$ increases the correlation of the samples. Therefore, based on Haario et al. (2001), Papaioannou et al. (2015) proposed an adaptive scheme for tuning $\rho_{k}$ in such a way that the acceptance probability is close to the optimal value of 0.44 ; this scheme is shown in Algorithm 2 and its details can be found in Papaioannou et al. (2015). It is worth mentioning that the CS algorithm can be regarded as a particular case of the MCMC sampler with preconditioned Crank-Nicolson proposal presented in Cotter et al. (2013), $q(\mathbf{u} \mid \mathbf{v})=\mathrm{N}\left(\mathbf{u} \mid \sqrt{1-\sigma^{2}} \mathbf{v}, \sigma^{2} \mathbf{\Sigma}\right), \boldsymbol{\Sigma}=\boldsymbol{I}$ and $\sigma \in[0,1]$.

\section{Proposed method: estimation of $\boldsymbol{P}_{\mathrm{f}}$ and $\overline{\boldsymbol{P}_{\mathrm{f}}}$ based on the $\mathscr{U} \rightarrow \Omega$ transformation}

From the foregoing discussion, we learned that the estimation of the bounds on the probability of failure when the input variables are expressed as probability boxes, possibility distributions, cumulative distribution functions or intervals, can be performed using random set theory. In this way, the calculation of the bounds of $P_{\mathrm{f}}$ is expressed as two separate probability of failure estimations, namely equations (9). These equations use as LSFs $g, \bar{g}: \Omega \rightarrow \mathbb{R}$, which are functions of the dependent variables $\bar{\alpha} \in \Omega$.

Many of the methods used to estimate the probability of failure, operate in the standard Gaussian space $\mathscr{U} \subseteq \mathbb{R}^{d}$; in consequence, it is required to transform the set of dependent vari- $\overline{\text { Algorithm } 2 \text { Adaptive conditional sampling for subset simula- }}$ tion (Papaioannou et al. (2015)).

\section{Input:}

1: Limit state function $G: \mathscr{U} \rightarrow \mathbb{R} \longrightarrow$ Seeds $\tilde{\boldsymbol{u}}_{j} \triangleright$ Intermediate threshold level $b_{j}$

2: Number of seeds $N_{s}=N p_{0} \quad$ Number of chains $N_{c}=1 / p_{0}$

3: Probability of adaptation $p_{a} \in[0.1,0.2]$

4: Number of chains for adaptation $N_{a}=N_{s} p_{a}$ ( $N_{a}$ is natural number)

5: Optimal acceptance rate $a^{*}=0.44 \longrightarrow$ Initial scaling parameter $\lambda_{0}=0.6$ Procedure:

6: Create three empty lists $\boldsymbol{u}_{j+1}, \boldsymbol{h}_{j+1}$ and $\boldsymbol{a}_{j+1}$

7: for $k=1$ to $d$ do

8: $\quad \tilde{\mu}_{j}^{(k)}=\frac{1}{N_{s}} \sum_{i=1}^{N_{s}} \tilde{u}_{j, i}^{(k)}$ and $\tilde{\sigma}_{j}^{(k)}=\sqrt{\frac{1}{N_{s}-1} \sum_{i=1}^{N_{s}}\left(\tilde{u}_{j, i}^{(k)}-\tilde{\mu}_{j}^{(k)}\right)^{2}}$ (estimate $\tilde{\sigma}_{j}$ from the seeds)

9: $\quad$ Set $\sigma_{k}=\min \left(1, \lambda_{0} \tilde{\sigma}_{j}^{(k)}\right)$ and $\rho_{k}=\sqrt{1-\sigma_{k}^{2}}$ (initial correlation parameter)

10: end for

11: Shuffle the seeds in $\tilde{\boldsymbol{u}}_{j}$ in order to avoid bias

12: Create the empty list $\overline{\boldsymbol{a}}$ (to store the acceptance/rejection values up until adaptation)

13: for $i=1$ to $N_{s}$ do

14: Create the empty lists $\boldsymbol{u} \boldsymbol{u}, \boldsymbol{h} \boldsymbol{h}$ and $\boldsymbol{a} \boldsymbol{a}$

15: $\quad$ Set $\boldsymbol{u} \boldsymbol{u}_{1}=\tilde{\boldsymbol{u}}_{j, i}$ (take the $i$-th seed)

16: Set $h h_{1}=G\left(\boldsymbol{u} \boldsymbol{u}_{1}\right)$ (compute the limit state value)

17: $\quad$ Set $a a_{1}=1$ (the seed is accepted by default)

18: for $p=1$ to $N_{c}-1$ do

19: $\quad$ for $k=1$ to $d$ do

20: $\quad$ Sample the candidate state $u_{k}^{*} \sim \mathrm{N}\left(\rho_{k} u u_{p}^{(k)}, \sigma_{k}^{2}\right)$

21: end for

22: $\quad$ Set $s=G\left(\boldsymbol{u}^{*}\right)$ (evaluate limit state function)

23: $\quad$ if $s \leq b_{j}$ then

24: $\quad$ Set $\boldsymbol{u} \boldsymbol{u}_{p+1}=\boldsymbol{u}^{*}, h h_{p+1}=s$ and $a a_{p+1}=1$ (acceptance)

25: $\quad$ else

26: $\quad$ Set $\boldsymbol{u} \boldsymbol{u}_{p+1}=\boldsymbol{u} \boldsymbol{u}_{p}, h h_{p+1}=h h_{p}$ and $a a_{p+1}=0$ (rejection)

27: $\quad$ end if

28: $\quad$ end for

29: Append the previous $N_{c}$ samples in $\boldsymbol{u} \boldsymbol{u}$ to the list $\boldsymbol{u}_{j+1}$

30: Append the previous $N_{c}$ limit state values $\boldsymbol{h} \boldsymbol{h}$ to the list $\boldsymbol{h}_{j+1}$

31: Append the previous $N_{c}$ acceptance/rejection values $\boldsymbol{a} \boldsymbol{a}$ to the lists $\boldsymbol{a}_{j+1}$ and $\overline{\boldsymbol{a}}$

32: $\quad$ if $\bmod \left(i, N_{a}\right)==0$ then

33: $\quad$ Set $t=$ floor $\left(i / N_{a}\right)$

34: $\quad$ Set $\hat{a}_{t}$ : Mean of the elements in the list $\overline{\boldsymbol{a}}$ (average acceptance rate)

35: $\quad$ Set $\lambda_{t}=\exp \left[\ln \left(\lambda_{t-1}\right)+\left(\hat{a}_{t}-a^{*}\right) / \sqrt{t}\right]$

36: $\quad$ for $k=1$ to $d$ do

37: $\quad \operatorname{Set} \sigma_{k}=\min \left(1, \lambda_{t} \tilde{\sigma}_{j}^{(k)}\right)$ and $\rho_{k}=\sqrt{1-\sigma_{k}^{2}}$ (adapt correlation parameter)

38: $\quad$ end for

39: $\quad$ Empty the list $\overline{\boldsymbol{a}}$

40: end if

41: end for

42: Output: $\boldsymbol{u}_{j+1}, \boldsymbol{h}_{j+1}$ and $\lambda_{t}$ (to be used as $\lambda_{0}$ in the next level)

ables in $\Omega$ to a set of independent variables in $\mathscr{U}$. This isoprobabilistic transformation can be easily performed using a bijective function $T$ that maps a dependent point in $\alpha \in \Omega$ according to the copula $C$ to an independent point in $[0,1]^{d}$, as explained in Ditlevsen and Madsen (1996, Chapter 7) and Lemaire et al. (2009, Chapter 4), so that $\boldsymbol{\alpha}=T^{-1}(\boldsymbol{\Phi}(\boldsymbol{u})$ ) and thus integrals (9) become,

$$
\underline{P_{\mathrm{f}}}=\int_{\mathscr{U}} \mathbb{I}[\bar{G}(\boldsymbol{u}) \leq 0] \mathrm{d} \Phi_{\boldsymbol{U}}(\boldsymbol{u}) \quad \overline{P_{\mathrm{f}}}=\int_{\mathscr{U}} \mathbb{I}[\underline{G}(\boldsymbol{u}) \leq 0] \mathrm{d} \Phi_{\boldsymbol{U}}(\boldsymbol{u})
$$


where, the LSFs $\underline{G}, \bar{G}: \mathscr{U} \rightarrow \mathbb{R}$ (see Figure 1c) are given by

$$
\underline{G}(\boldsymbol{u})=\underline{g}\left(T^{-1}(\boldsymbol{\Phi}(\boldsymbol{u}))\right) \quad \text { and } \quad \bar{G}(\boldsymbol{u})=\bar{g}\left(T^{-1}(\boldsymbol{\Phi}(\boldsymbol{u}))\right) ;
$$

here, $\Phi_{\boldsymbol{U}}$ stands for the standard Gaussian joint CDF, $\boldsymbol{\Phi}(\boldsymbol{u})=$ $\left[\Phi\left(u_{1}\right), \Phi\left(u_{2}\right), \ldots, \Phi\left(u_{d}\right)\right]$, and $\Phi$ is the unidimensional standard Gaussian CDF. Basically, the operation $\boldsymbol{\Phi}(\boldsymbol{u})$ transforms the standard Gaussian variables in $\mathscr{U}$ to the independent space $[0,1]^{d}$ and the inverse transformation $T^{-1}$ maps an independent point in $[0,1]^{d}$ to a dependent one in $\Omega$ according to the copula $C$. This last transformation can be performed using for instance the Rosenblatt transform $T_{\text {Ros }}$, as explained in Section 2.3.

We can solve both reliability problems (11) using for example, FORM, importance sampling, line sampling, the cross-entropy method or even SuS. In the following section, we will illustrate the proposed approach using SuS. Note however that this method is different from the one in Alvarez et al. (2014); in that paper, we suggested an algorithm that modifies the MCMC procedure of Au and Beck (2003), so that it samples directly in the $\Omega$-space instead of the $\mathscr{U}$-space, thus the transformation of equation (12) is not required. That method was only applicable to $\mathrm{SuS}$, while the present approach is applicable to any method that estimates the probability of failure in the standard Gaussian space, as is the common practice in reliability analysis.

\section{Numerical examples}

Let us illustrate the proposed approach with three numerical examples that are solved using Monte Carlo simulation and subset simulation with both adaptive conditional sampling (ACS) and the modified Metropolis algorithm (MMA). For the SuS method, the image of $N=1000$ focal elements are calculated per level and the results (probability bounds and associated coefficients of variation (c.o.v.)) are computed as an average of 50 independent runs with a conditional probability value of $p_{0}=0.1$. For ACS, the probability of adaptation $p_{a}$ is set to 0.1 and for MMA, a uniform proposal with width 2 is used.

Notice that in a traditional full-probabilistic setting, one LSF evaluation will suffice to check whether the sample belongs to the failure region or not. However, in the random set case, the application of the extension principle is necessary to obtain the lower and upper LSFs. This implies additional model evaluations per focal element due to the optimization problem. Therefore, in all the examples, the image of the focal element $\Gamma(\boldsymbol{\alpha})$ through the LSF $g$ was found by solving the constrained optimization problems in equations (6) using an interior-point algorithm (Potra and Wright, 2000). We are using this method instead of others with a lower computational cost (e.g. the vertex method), because this guarantees that the images of the focal elements are precisely computed in all examples.

\subsection{Example 1: a two-dimensional function}

Consider the $\operatorname{LSF} g(\boldsymbol{x})=7-x_{1}^{2}+x_{2}$. The input variables $X_{1}$ and $X_{2}$ are respectively modeled as the distributional p-boxes
$\mathrm{N}([-1,2], 1)$ and $\mathrm{N}([-2,1], 2)$. As described before, these distributional p-boxes must be converted to distribution-free $\mathrm{p}$ boxes in order to operate with random set theory. Moreover, both input variables are assumed to be independent and therefore a product copula relates them, $C(\boldsymbol{\alpha})=\alpha_{1} \alpha_{2}$.

The computation of the bounds of the probability of failure is given by the integrals (11). After applying the MCS estimators (10) using $1 \times 10^{6}$ focal element evaluations for the lower bound and $1 \times 10^{4}$ for the upper bound, the estimates of the probability bounds are $\left[\hat{P}_{\mathrm{f}}, \hat{P_{\mathrm{f}}}\right]=\left[2.590 \times 10^{-4}, 0.503\right]$. Using the optimization method, the determination of the image of each focal element required in average 33.82 and 34.21 LSF evaluations per element for the lower and upper bounds, respectively. Take into account that the procedure right after equation (10) was employed in order to reduce the number of focal element evaluations in the estimation of $P_{\mathrm{f}}$. In this case, 4970 samples did not comply with $\underline{G}(\boldsymbol{u}) \leq 0$, and therefore only 995030 focal element evaluations were required in the estimation of $P_{\mathrm{f}}$ by means of MCS.

In $\mathrm{SuS}$ with ACS the lower bound is computed with $\bar{G}(\boldsymbol{u})$ as LSF, in this case the intermediate levels are $b_{j}=[5.305,3.097$, $1.271,0]$, obtaining the estimate $\underline{\hat{P}_{\mathrm{f}}}=2.532 \times 10^{-4}$ with c.o.v. $=$ 0.3132 . Then, the upper bound is calculated with the LSF $\underline{G}(\boldsymbol{u})$, in this case there are no intermediate levels $b_{j}=0$, and the estimate is $\hat{P_{\mathrm{f}}}=0.500$ with c.o.v. $=0.0301$. This result is basically the probability of failure estimated by MCS, and hence, SuS is especially useful in the estimation of the lower probability bound. For comparison, the same computation is carried out using the classical MMA method. In this case, for the lower bound the intermediate levels are $b_{j}=[5.329,3.1093,1.269,0]$ to obtain the estimate $\hat{P}_{\mathrm{f}}=2.575 \times 10^{-4}$ with c.o.v. $=0.4265$. For the upper bound, no intermediate levels are required $b_{j}=0$, and the estimate is $\hat{\overrightarrow{P_{\mathrm{f}}}}=0.49911$ with c.o.v. $=0.0361$. Finally, during the estimation of the lower bound, the calculation of the image of each focal element within $\mathrm{SuS}$ required in average 33.82, 33.34, 32.07 and 29.30 LSF evaluations at each intermediate threshold level.

The advantage of this example lies in the possibility to illustrate how the samples are distributed in the three working spaces, that is: $i)$ the original space of basic random variables $\mathscr{X}$, which contains the focal elements of the random set as $d$-boxes; ii) the domain of definition of the copulas $\Omega$, which allows to model the dependence between the input variables and specifies the focal elements as the points $\boldsymbol{\alpha}_{i}$; and iii) the space of standard Gaussian random variables $\mathscr{U}$, where the structural reliability problem is solved. Figure 2 shows the distribution of the SuS+ACS samples for the computation of the lower bound (three intermediate levels) and of the upper bound (no intermediate levels). Observe that in the $\mathscr{X}$-space, the focal elements of the random sets are defined as two-dimensional boxes; here it is also depicted the failure surface $g(\boldsymbol{x})=0$. For the other spaces is difficult to represent the shape of the corresponding lower and upper LSFs since it would require the evaluation of a considerable amount of samples. Thus, the failure regions can 
Table 1: Input variables of the problem analyzed in Example 2.

\begin{tabular}{lcl}
\hline \hline Variable & Units & Modeled as \\
\hline \hline$X_{1}\left(F_{1}\right)$ & $\mathrm{kN}$ & $\mathrm{N}\left(2,0.2^{2}\right)$ \\
$X_{2}\left(F_{2}\right)$ & $\mathrm{kN}$ & $\mathrm{N}\left(3,0.3^{2}\right)$ \\
$X_{3}(P)$ & $\mathrm{kN}$ & $\mathrm{G}([11.9,12.1],[1.19,1.21])$ \\
$X_{4}(T)$ & $\mathrm{N} \cdot \mathrm{m}$ & $\mathrm{N}\left(90,\left[8.95^{2}, 9.05^{2}\right]\right)$ \\
$X_{5}\left(\sigma_{y}\right)$ & $\mathrm{MPa}$ & $\mathrm{LN}\left(220,22^{2}\right)$ \\
$X_{6}(t)$ & $\mathrm{mm}$ & fuzzy trapezoidal $(2.8,2.9,3.1,3.2)$ \\
$X_{7}(d)$ & $\mathrm{mm}$ & fuzzy triangular $(41.8,42,42.2)$ \\
$X_{8}\left(L_{1}\right)$ & $\mathrm{mm}$ & interval $(119.75,120.25)$ \\
$X_{9}\left(L_{2}\right)$ & $\mathrm{mm}$ & interval $(59.75,60.25)$ \\
$X_{10}\left(\theta_{1}\right)$ & degrees & interval $(19,21)$ \\
$X_{11}\left(\theta_{2}\right)$ & degrees & interval $(30,35)$ \\
\hline \hline
\end{tabular}

be differentiated from the safe regions by the distribution of the samples. Note also that all of the "failure boxes" (in red) associated to the estimation of $P_{\mathrm{f}}$ were contained in the failure region $F$, while none of the "failure boxes" associated to the estimation of $\overline{P_{\mathrm{f}}}$ were completely contained in the safe region $S$.

\subsection{Example 2: a cantilever tube}

Let us consider a modified version of the example proposed in Du (2008), which deals with a cantilever tube beam of diameter $d$ and thickness $t$, sketched in Figure 3. The beam is subjected to a torsional moment $T$, external lateral forces $F_{1}, F_{2}$ and axial force $P$.

The failure of the beam occurs when the maximum von Mises stress on the top surface of the tube at the support, $\sigma_{\max }=$ $\sqrt{\sigma_{x}^{2}+3 \tau_{x z}^{2}}$, is larger than the yield strength $\sigma_{y}$ of the material; in this sense, the LSF is defined as the difference $g(\boldsymbol{x})=$ $\sigma_{y}-\sigma_{\max }$, where

$$
\sigma_{x}=\frac{P+F_{1} \sin \theta_{1}+F_{2} \sin \theta_{2}}{A}+\frac{M d}{2 I}
$$

stands for the normal stress produced by the axial and lateral forces and bending moments, $\tau_{x z}=(T d) /(4 I)$ is the shear stress produced by the torsion at the support, $A=\pi\left(d^{2}-(d-2 t)^{2}\right) / 4$ is the cross-sectional area, $I=\pi\left(d^{4}-(d-2 t)^{4}\right) / 64$ is the second moment of inertia, and $M=F_{1} L_{1} \cos \theta_{1}+F_{2} L_{2} \cos \theta_{2}$ represents the bending moment in the beam.

The uncertain input variables to the system are listed in Table 1. Here, $X_{1}, X_{2}$ and $X_{5}$ are represented as CDFs, $X_{3}$ and $X_{4}$ are modeled as distributional p-boxes converted to distribution-free p-boxes, $X_{6}$ and $X_{7}$ are possibility distributions, and variables $X_{8}, X_{9}, X_{10}$ and $X_{11}$ are typified as intervals. In Table 1 , the nomenclature $\mathrm{N}\left(\mu, \sigma^{2}\right)$ expresses the Gaussian CDF with mean $\mu$ and variance $\sigma^{2}, \mathrm{G}(\mu, \sigma)$ symbolizes a Gumbel (Type I extreme value) $\operatorname{CDF} G(x ; \mu, \sigma)=\exp (-\exp ((x-\mu) / \sigma))$ with location parameter $\mu$ and scale parameter $\sigma$, and $\operatorname{LN}(m, v)$ stands for a lognormal CDF with mean $m$ and variance $v$.
Furthermore, we will suppose that variables $X_{5}$ to $X_{11}$ are independent and consequently they are related through a product copula $C(\alpha):=\prod_{i=1}^{\operatorname{dim}(\alpha)} \alpha_{i}$, while variables $X_{1}$ to $X_{4}$ are related through a Frank copula

$$
C_{\text {Frank }}(\boldsymbol{\alpha} ; \delta):=\psi^{-1}\left(\sum_{i=1}^{\operatorname{dim}(\boldsymbol{\alpha})} \psi\left(\alpha_{i} ; \delta\right) ; \delta\right)
$$

with $\psi(t ; \delta)=-\ln \left(\frac{\exp (-\delta t)-1}{\exp (-\delta)-1}\right), \quad \psi^{-1}(t ; \delta) \quad=$ $-\frac{1}{\delta} \ln (1+\exp (-t)(\exp (-\delta)-1))$ and parameter $\delta \in \mathbb{R} \backslash 0$ set to be equal to $\delta=10$. In consequence, the copula that relates all implied variables is

$$
C(\boldsymbol{\alpha})=C_{\text {Frank }}\left(\left[\alpha_{1}, \alpha_{2}, \alpha_{3}, \alpha_{4}\right] ; 10\right) \cdot \prod_{i=5}^{11} \alpha_{i} .
$$

As in the previous example, the failure probability bounds are initially estimated by MCS. In this case, $N=1 \times 10^{6}$ and $N=1 \times 10^{5}$ focal elements are used for the estimation of the lower and upper bounds. The determination of the image of each focal element using the optimization method required in average 112.3 and 107.3 LSF evaluations per element for the lower and upper bounds, respectively. The results are $\left[\hat{P}_{\mathrm{f}}, \hat{\overline{P_{\mathrm{f}}}}\right]=\left[5.230 \times 10^{-4}, 1.003 \times 10^{-2}\right]$. Again, by applying the procedure right after equation (10), the number of focal element evaluations in the estimation of $P_{\mathrm{f}}$ was reduced. In this case, 98997 samples did not comply with $\underline{G}(\boldsymbol{u}) \leq 0$, and thus 901003 focal element evaluations were required in the estimation of $P_{\mathrm{f}}$ by means of MCS.

In SuS with ACS, the estimation of the lower bound required four intermediate levels and the estimate is $\hat{P}_{\mathrm{f}}=5.136 \times 10^{-4}$ with c.o.v. $=0.2429$. For the upper bound three intermediate level are reached, giving the estimate $\hat{P_{\mathrm{f}}}=8.588 \times 10^{-3}$ with c.o.v. $=0.1039$. Again, $\mathrm{SuS}$ runs are also performed with MMA. For the lower bound, the estimate is $\hat{P}_{\mathrm{f}}=5.244 \times 10^{-4}$ with c.o.v. $=0.2916$. For the upper bound, the estimate is $\hat{\overline{P_{\mathrm{f}}}}=8.301 \times 10^{-3}$ with c.o.v. $=0.1382$. Moreover, in the estimation the lower bound, the calculation of the image of each focal element within SuS required in average 112.29, 113.04, 113.26 and 113.56 LSF evaluations at each intermediate threshold level; and for the upper bound, the calculation of the image of each focal element required in average 107.16, 107.50 and 107.96 LSF evaluations at each intermediate level.

In this example the failure threshold level $\sigma_{y}$ is also considered uncertain, thus, it is not feasible to generate an excursion probability plot to represent the evolution of the response quantity $\sigma_{\text {max }}$ (threshold levels) with respect to the probability of failure. However, it is possible to illustrate how the lower and upper LSFs values $h$ evolve with the probability; Figure 4 shows this evolution using the MCS and SuS+ACS methods. For each bound, the average of $50 \mathrm{SuS}$ runs shows a good agreement with the reference MCS estimation. Furthermore, Figure 5 shows a comparison between the ACS and MMA methods, in terms of the coefficients of variation of the probability estimates. 

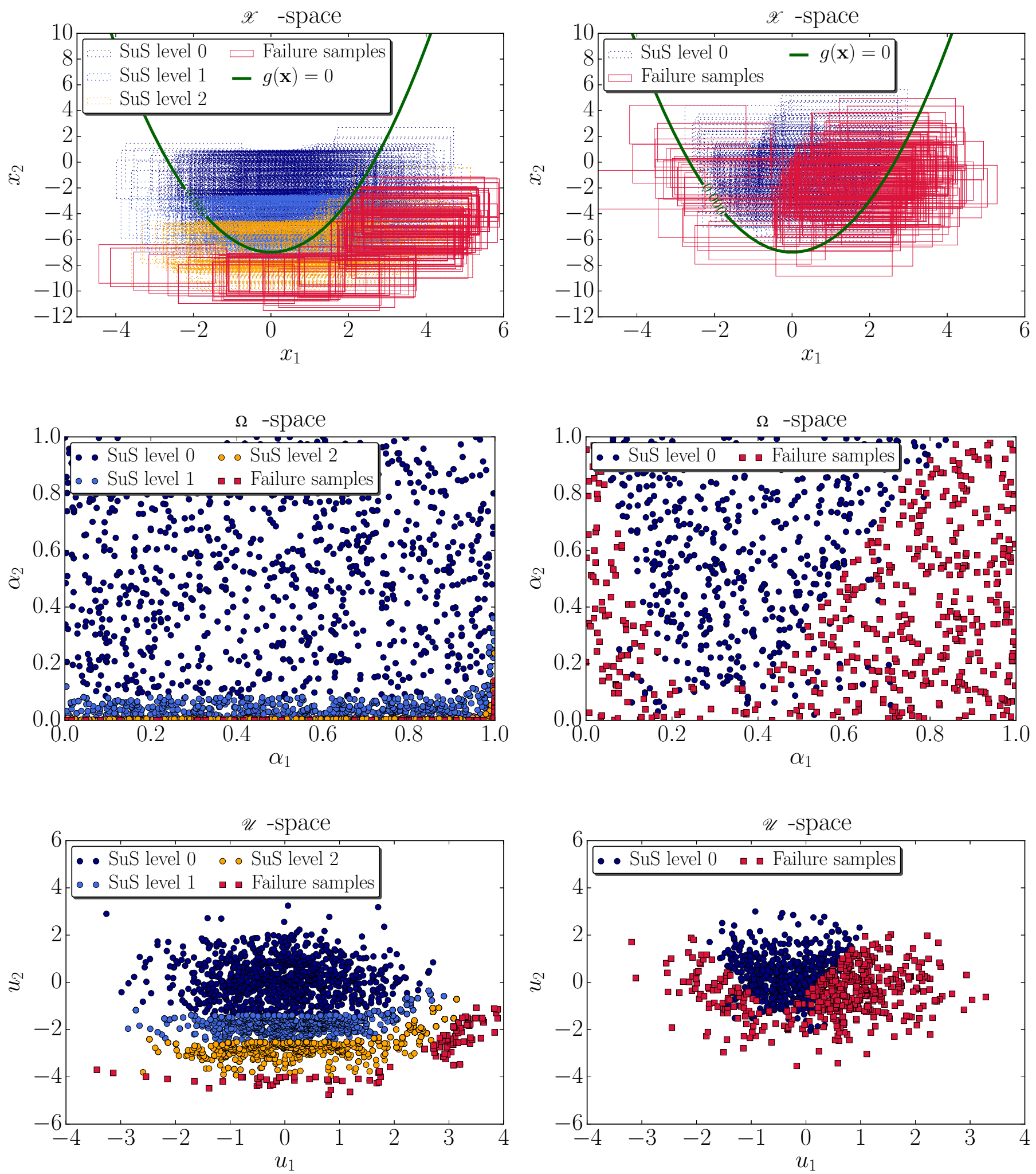

Figure 2: SuS evolution in the spaces $\mathscr{X}, \Omega$ and $\mathscr{U}$ for the computation of lower bound $\hat{\hat{P}}_{\mathrm{f}}$ (left) and upper bound (right) $\hat{\bar{P}_{\mathrm{f}}}$ of the Example 1 . 


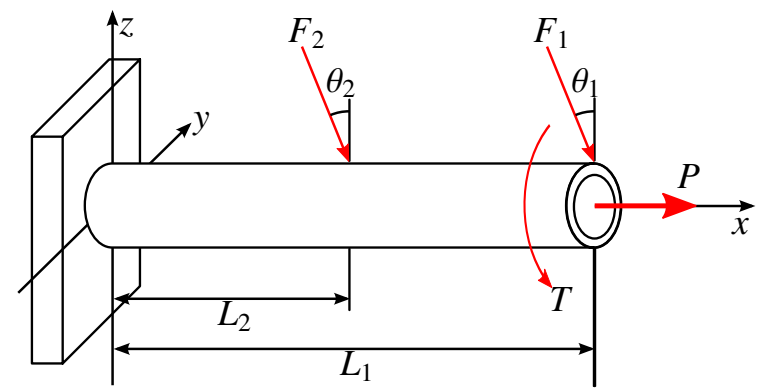

Figure 3: Structure considered in the Example 2.

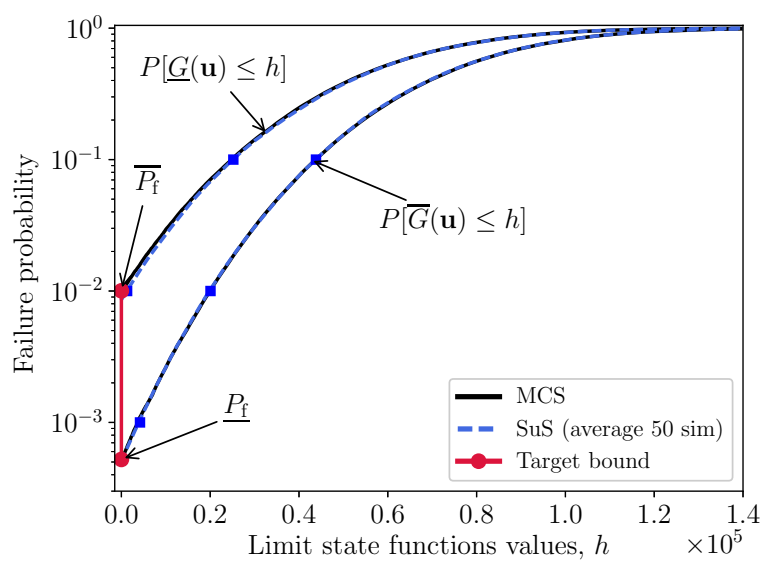

Figure 4: Limit state function evolution: estimate of the probability of failure bounds for the Example 2.

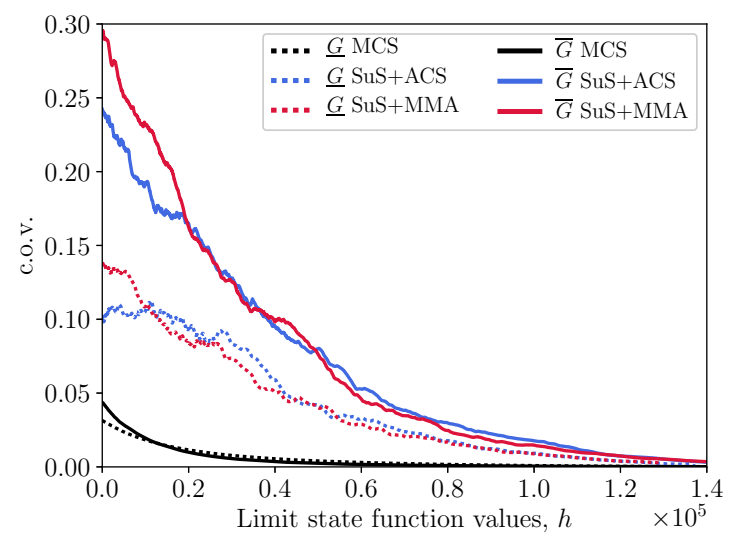

Figure 5: Limit state function values vs. coefficient of variation, for MCS and SuS with the ACS and MMA methods (Example 2).

\subsection{Example 3: a six-bar truss}

Consider the six-bar steel truss shown in Figure 6. The structural failure occurs when the displacement of upper-right node in the positive $x$-direction $\Delta$ (drift) exceeds $1.25 \mathrm{~mm}$; therefore, the LSF is defined as $g(\boldsymbol{x})=1.25-\Delta(\boldsymbol{x})$. Here, $\Delta$ is measured in millimeters, the Young modulus is $E=210 \mathrm{GPa}$, the external cross-sectional areas of the bars $\left(A_{1}, \ldots, A_{4}\right)$ are described by random variables, and the applied loads $\left(P_{1}, \ldots, P_{4}\right)$ and the internal cross-sectional areas $\left(A_{5}\right.$ and $\left.A_{6}\right)$ are described by dis-
Table 2: Input variables of the problem analyzed in the Example 3.

\begin{tabular}{lll}
\hline \hline Variable & Units & Modeled as \\
\hline \hline$X_{1}, \ldots, X_{4}$ & $\left(P_{1}, \ldots, P_{4}: \mathrm{kN}\right)$ & $\mathrm{N}\left([35,37], 5^{2}\right)$ \\
$X_{5}, \ldots, X_{8}$ & $\left(A_{1}, \ldots, A_{4}: \mathrm{cm}^{2}\right)$ & $\mathrm{LN}\left(10,0.01^{2}\right)$ \\
$X_{9}, X_{10}$ & $\left(A_{5}, A_{6}: \mathrm{cm}^{2}\right)$ & $\mathrm{U}([9.2,10.3],[10.8,12.1])$ \\
\hline \hline
\end{tabular}

tributional p-boxes, as specified in Table 2.

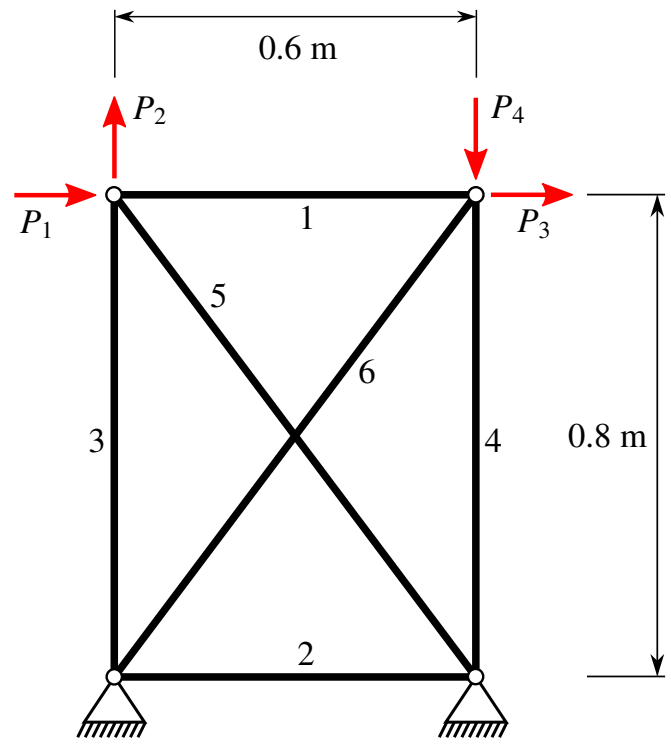

Figure 6: Six-bar truss considered in the Example 3.

In this example, the dependence between variables $P_{1}$ and $P_{2}$ is given by a Frank copula with $\delta=5$, the dependence between variables $P_{3}$ and $P_{4}$ is also given by a two-dimensional Frank copula with $\delta=5$; and variables $A_{1}, \ldots, A_{6}$ are related by a Gaussian copula $C_{\text {Gauss }}\left(\left[\alpha_{5}, \ldots, \alpha_{10}\right] ; \boldsymbol{R}_{G}\right)$ with correlation matrix

$$
\boldsymbol{R}_{G}=\left(\begin{array}{cccccc}
1 & 0.9 & 0.3 & 0.3 & 0.3 & 0.3 \\
0.9 & 1 & 0.3 & 0.3 & 0.3 & 0.3 \\
0.3 & 0.3 & 1 & 0.9 & 0.3 & 0.3 \\
0.3 & 0.3 & 0.9 & 1 & 0.3 & 0.3 \\
0.3 & 0.3 & 0.3 & 0.3 & 1 & 0.9 \\
0.3 & 0.3 & 0.3 & 0.3 & 0.9 & 1
\end{array}\right)
$$

The Gaussian copula with correlation matrix $\boldsymbol{R}_{G} \in[-1,1]^{d \times d}$ is given by

$$
C_{\text {Gauss }}\left(\alpha ; \boldsymbol{R}_{G}\right)=\Phi_{\boldsymbol{R}_{G}}\left(\Phi^{-1}\left(\alpha_{1}\right), \ldots, \Phi^{-1}\left(\alpha_{d}\right)\right)
$$

where, $\Phi^{-1}$ is the quantile function of a standard Gaussian CDF and $\Phi_{\boldsymbol{R}_{G}}$ is the joint CDF of a multivariate Gaussian distribution with zero mean vector and covariance matrix equal to $\boldsymbol{R}_{G}$. Since the groups of variables $\left\{P_{1}, P_{2}\right\},\left\{P_{3}, P_{4}\right\}$ and $\left\{A_{1}, \ldots, A_{6}\right\}$ are independent, the variables are related by the copula:

$$
\begin{aligned}
C(\boldsymbol{\alpha})= & C_{\text {Frank }}\left(\left[\alpha_{1}, \alpha_{2}\right] ; 5\right) \cdot C_{\text {Frank }}\left(\left[\alpha_{3}, \alpha_{4}\right] ; 5\right) \\
& \cdot C_{\text {Gauss }}\left(\left[\alpha_{5}, \ldots, \alpha_{10}\right] ; \boldsymbol{R}_{G}\right) .
\end{aligned}
$$


On the one hand, integrals (11) are estimated using MCS, obtaining the failure probability bounds $\underline{\hat{P}_{\mathrm{f}}}=2.580 \times 10^{-5}$ and $\hat{\overline{P_{\mathrm{f}}}}=1.670 \times 10^{-3}$; this estimation was computed by calculating the image of $1 \times 10^{7}$ and $1 \times 10^{5}$ focal elements, respectively. As in the previous examples, by applying the ideas right after equation (10), the number of focal element evaluations in the estimation of $P_{\mathrm{f}}$ can be reduced. In this case, 99833 samples did not comply with $\underline{G}(\boldsymbol{u}) \leq 0$, and therefore 9900167 focal element evaluations were required in the estimation of $P_{\mathrm{f}}$ by means of MCS. Using the optimization method, the determination of the image of each focal element required in average 206.91 and 255.01 samples per element, for the lower and upper bounds.

On the other hand, the average $\mathrm{SuS}+\mathrm{ACS}$ runs estimated five intermediate levels $b_{j}=[0.959,1.065,1.145,1.212,1.25]$ for the calculation of the lower bound to obtain $\hat{P}_{\mathrm{f}}=2.523 \times 10^{-5}$ with c.o.v. $=0.3325$; and for the upper bound, three intermediate levels are reached $b_{j}=[1.068,1.181,1.25]$ giving the estimate $\hat{\overline{P_{\mathrm{f}}}}=1.609 \times 10^{-3}$ with c.o.v. $=0.2026$. The same computation is carried out using the MMA, providing the following results: for the lower bound, the intermediate levels are $b_{j}=[0.989,1.096,1.176,1.242,1.25]$ to obtain the estimate $\underline{\hat{P}_{\mathrm{f}}}=2.565 \times 10^{-5}$ with c.o.v. $=0.3979$; and for the upper bound, the intermediate levels are $b_{j}=[1.066,1.181,1.25]$ to estimate $\hat{\overline{P_{\mathrm{f}}}}=1.664 \times 10^{-3}$ with c.o.v. $=0.2454$. During the estimation the lower bound, the calculation of the image of each focal element in SuS required in average 206.65, 203.43, 201.28, 199.61 and 198.35 LSF evaluations at each intermediate threshold level; and for the upper bound, the calculation of the image of each focal element required in average 254.91,254.39 and 253.61 LSF evaluations at each intermediate level.

In contrast to Example 2, the probability excursion can be estimated since the failure threshold level is assumed to be constant $(1.25 \mathrm{~mm})$. Figure 7 shows a plot of the lower and upper probabilities of failure for each drift level $\Delta(\boldsymbol{x})$. As in Example 2, it is seen that the results by MCS and SuS+ACS are in agreement. Observe that with the average runs of subset simulation we can obtain the bounds of the probability of failure for each demand level up to the largest one considered. Additionally, Figure 8 presents a comparison between the ACS and MMA methods, in terms of the evolution of the drift values and the coefficients of variation of the failure probability estimates.

\section{Discussion of results}

The problem of finding the lower and upper bounds of the probability of failure has been expressed as a reliability problem that models the dependence by means of copulas. However, since many of the methods for the estimation of the probability of failure have been designed to operate in the standard Gaussian space, it is required to use a a suitable isoprobabilistic transformation to map any copula to such space.

The proposed examples confirm that subset simulation is especially useful in the estimation of those bounds. This is mainly

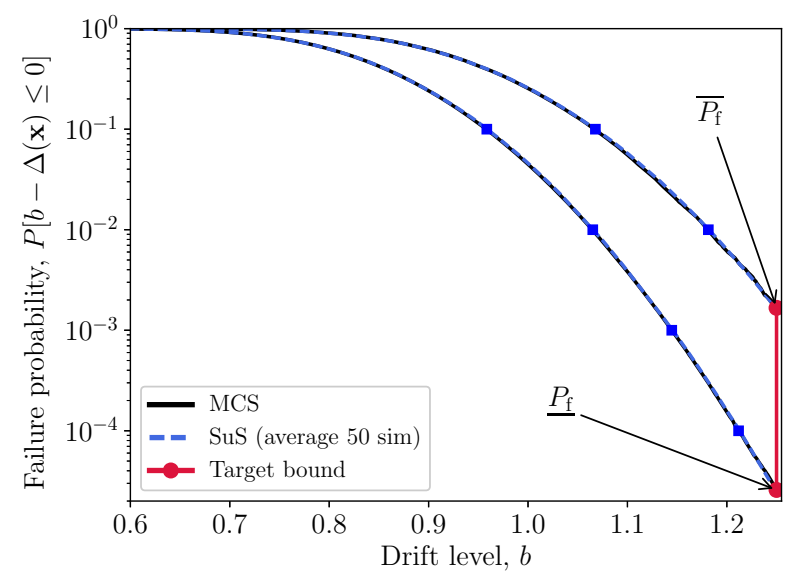

Figure 7: Probability excursion: estimate of the probability of failure bounds for the Example 3.

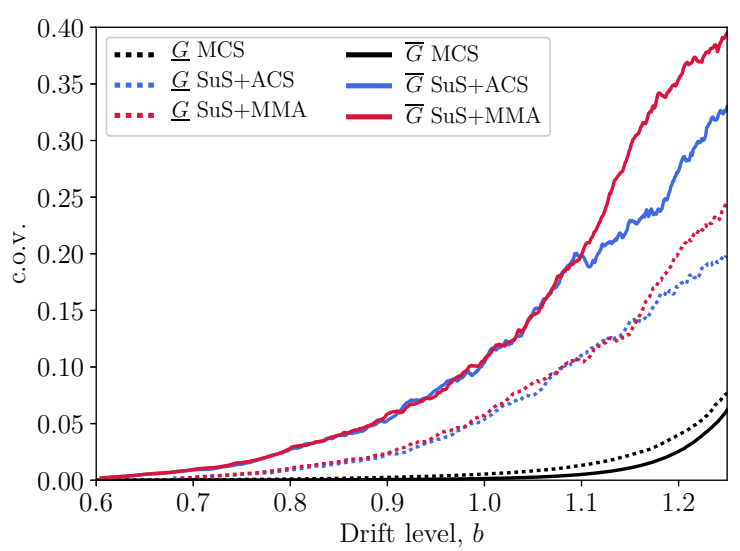

Figure 8: Drift levels vs. coefficient of variation, for MCS and SuS with the ACS and MMA methods (Example 3).

related to the fact that the method results in a considerable reduction of the computational effort, and at the same time, gives results that are in a good agreement with the reference Monte Carlo solution. Perhaps the main issue of subset simulation lies in the variability of the simulation results which generally produces large coefficients of variation. We assessed the performance of two well-known MCMC samplers for subset simulation, namely, the modified metropolis algorithm and the adaptive conditional sampler. For all the examples, the ACS algorithm provides lower coefficients of variation compared to the MMA. Nevertheless, the estimation of the failure probability bounds by subset simulation using either MMA or ACS provides results that in average are in agreement with the reference Monte Carlo solution.

Since the calculation of $\underline{G}(\boldsymbol{u})$ and $\bar{G}(\boldsymbol{u})$ requires the solution of two optimization problems, many LSF evaluations per focal element are required. Therefore, the average number of LSF calls is also computed as a measure of the computational cost. Particularly, for most of the examples using SuS, the number of LSF calls decreased with each intermediate level as the failure 
space is sequentially explored.

When using imprecise probability methods, small intervals in the definition of the parameters of the input variables, may lead to wide bounds on the estimated probability of failure. This should not be taken as an argument against these techniques, though; on the contrary, this indicates that it is very dangerous to assume unique values on the parameters of the input variables, especially in the case of scarce data or vague information, in order to obtain a point estimate of the probability of failure. Naturally, the width of those intervals is expected to narrow when additional information is available. In those cases, sensitivity analysis methods can also be used to address the situation (see e.g. Alvarez (2009b); Patelli et al. (2014)).

As previously commented, Alvarez et al. (2017) devised a method to estimate the bounds of the probability of failure by dealing directly with distributional p-boxes, without recurring to the approximation of a distributional p-box as a distributionfree p-box. As a result, narrower bounds on the probability of failure are found, since the additional information included with the approximation is excluded in the calculation. For instance, when using the methods introduced in Alvarez et al. (2017), the bound on the probability of failure for the Example 1 is $\left[8.999 \times 10^{-3}, 0.431\right]$, which compared to the bound provided by random set theory (that is $\left[2.590 \times 10^{-4}, 0.503\right]$ ) produces a tightening of around $16 \%$.

Finally, the results are summarized in Table 3 for MCS, $\mathrm{SuS}+\mathrm{ACS}$ and $\mathrm{SuS}+\mathrm{MMA}$, where the coefficients of variation for SuS were computed using 50 independent runs. The average number of LSF in SuS was computed along the simulation runs and also along the intermediate levels.

\section{Conclusions and open research problems}

An efficient approach for the reliability analysis of structures under uncertainty, in which the input variables are modeled using random set theory (that is, possibility distributions, intervals, CDFs, distribution-free p-boxes or even Dempster-Shafer structures) was presented. Each focal element that is sampled from the random set is modeled either as a point in a space $\Omega$, or as a $d$-dimensional box in the space of input variables $\mathscr{X}$. In the $\Omega$-space, a copula $C$ models the dependence between the input variables; also in $\Omega$ there exists two limit state functions $g$ and $\bar{g}$ that define the lower and upper inverses of the failure set, that is $F_{L P}$ and $F_{U P}$. The focal elements corresponding to $F_{L P}$ and $F_{U P}$ contribute to the evaluation of the lower and upper probability of failure, respectively. By mapping the limit state functions $\underline{g}$ and $\bar{g}$ to the standard Gaussian space, using an inverse isoprobabilistic transformation $T^{-1}$ (e.g. the inverse Rosenblatt transform) that maps an independent point in $[0,1]^{d}$ to a dependent one in $\Omega$ according to the copula $C$, we can easily estimate by any advanced Monte Carlo method, such as subset simulation, importance sampling, line sampling, or the cross-entropy method, among others, the lower and upper bounds on the probability of failure. Particularly, we illus- trated this approach using subset simulation with two different MCMC samplers, adaptive conditional sampling and modified Metropolis-Hastings. The numerical experiments demonstrated the solid foundation of this proposal, where a drastic reduction of the computational labor implied by plain Monte Carlo simulation was obtained for problems defined with uncertain input aleatory and/or epistemic distributions.

Some open research problems are the following: the proposed method reduces the number of focal element images that are needed in order to estimate $P_{\mathrm{f}}$ and $\overline{P_{\mathrm{f}}}$, however, the number of evaluations of the limit state function $g$ required in the calculation, although considerably reduced, still is a large number that should be diminished. A possible solution for this is to employ the proposed method with subset simulation together with response surfaces, such as, kriging or neural networks; see for example (Hurtado et al., 2000; Hurtado and Alvarez, 2001; Echard et al., 2011; Papadopoulos et al., 2013). Furthermore, it is important to mention that copulas solely model the dependence between the input variables and not within the focal elements. It might seem strange that a copula can contain the dependence information of two possibility distributions as input variables. However, take into account that many copulas are t-norms and many t-norms are copulas, i.e., copulas are the mathematical tool for combining CDFs and p-boxes, while tnorms are the mathematical tool for combining membership functions of fuzzy sets and possibility distributions. For further discussion on the relationship between copulas, p-boxes and Dempster-Shafer structures, see for example Ferson et al. (2004). Up to the author's knowledge, the use of copulas to model the dependence between p-boxes and possibility distributions is up to now an open problem in the context of the estimation of the probability of failure using random sets.

\section{Acknowledgements}

Financial support for the realization of the present research has been received from the Universidad Nacional de Colombia. The support is gratefully acknowledged.

The second author acknowledges the support from the Deutsche Forschungsgemeinschaft (DFG) through the TUM International Graduate School of Science and Engineering (IGSSE).

\section{References}

Alvarez, D. A., 2006. On the calculation of the bounds of probability of events using infinite random sets. International Journal of Approximate Reasoning 43, 241-267.

Alvarez, D. A., 2007. Infinite random sets and applications in uncertainty analysis. Ph.D. thesis, Arbeitsbereich für Technische Mathematik am Institut für Grundlagen der Bauingenieurwissenschaften. Leopold-FranzensUniversität Innsbruck, Innsbruck, Austria, available at https://sites . google.com/site/diegoandresalvarezmarin/RSthesis . pdf.

Alvarez, D. A., 2009a. A Monte Carlo-based method for the estimation of lower and upper probabilities of events using infinite random sets of indexable type. Fuzzy Sets and Systems 160, 384-401. 
Table 3: Probability bounds using Monte Carlo and subset simulation with ACS and MMA. The column "average LSF eval." reports the average number of LSF evaluations per focal element.

\begin{tabular}{|c|c|c|c|c|c|c|c|c|}
\hline & \multicolumn{8}{|c|}{ Example 1} \\
\hline & \multicolumn{4}{|c|}{ Lower bounds } & \multicolumn{4}{|c|}{ Upper bounds } \\
\hline & $N$ & $\begin{array}{l}\text { Average } \\
\text { LSF eval. }\end{array}$ & ${\hat{P_{\mathrm{f}}}}_{\underline{\mathrm{N}}}$ & c.o.v. & $N$ & $\begin{array}{l}\text { Average } \\
\text { LSF eval. }\end{array}$ & $\overline{\hat{P}_{\mathrm{f}}}$ & c.o.v. \\
\hline MCS & $1 \times 10^{6}$ & 33.8 & $2.590 \times 10^{-4}$ & - & $1 \times 10^{4}$ & 34.2 & 0.503 & - \\
\hline $\mathrm{SuS}+\mathrm{ACS}$ & $4 \times 10^{3}$ & 32.0 & $2.532 \times 10^{-4}$ & 0.313 & $1 \times 10^{3}$ & 33.8 & 0.500 & 0.030 \\
\hline SuS+MMA & $4 \times 10^{3}$ & 32.3 & $2.575 \times 10^{-4}$ & 0.427 & $1 \times 10^{3}$ & 34.1 & 0.499 & 0.036 \\
\hline
\end{tabular}

\begin{tabular}{|c|c|c|c|c|c|c|c|c|}
\hline \multirow[b]{4}{*}{ MCS } & \multicolumn{8}{|c|}{ Example 2} \\
\hline & \multicolumn{4}{|c|}{ Lower bounds } & \multicolumn{4}{|c|}{ Upper bounds } \\
\hline & $N$ & $\begin{array}{l}\text { Average } \\
\text { LSF eval. }\end{array}$ & $\underline{\hat{P}_{\mathrm{f}}}$ & c.o.v. & $N$ & $\begin{array}{l}\text { Average } \\
\text { LSF eval. }\end{array}$ & $\overline{\hat{P}_{\mathrm{f}}}$ & c.o.v. \\
\hline & $1 \times 10^{6}$ & 112.3 & $5.230 \times 10^{-4}$ & - & $1 \times 10^{5}$ & 107.3 & $1.003 \times 10^{-2}$ & - \\
\hline $\mathrm{SuS}+\mathrm{ACS}$ & $4 \times 10^{3}$ & 113.0 & $5.136 \times 10^{-4}$ & 0.243 & $3 \times 10^{3}$ & 107.5 & $8.588 \times 10^{-3}$ & 0.104 \\
\hline SuS+MMA & $4 \times 10^{3}$ & 113.1 & $5.244 \times 10^{-4}$ & 0.292 & $3 \times 10^{3}$ & 107.5 & $8.301 \times 10^{-3}$ & 0.138 \\
\hline
\end{tabular}

\begin{tabular}{|c|c|c|c|c|c|c|c|c|}
\hline \multirow[b]{4}{*}{ MCS } & \multicolumn{8}{|c|}{ Example 3} \\
\hline & \multicolumn{4}{|c|}{ Lower bounds } & \multicolumn{4}{|c|}{ Upper bounds } \\
\hline & $N$ & $\begin{array}{l}\text { Average } \\
\text { LSF eval. }\end{array}$ & $\underline{\hat{P}_{\mathrm{f}}}$ & c.o.v. & $N$ & $\begin{array}{l}\text { Average } \\
\text { LSF eval. }\end{array}$ & $\overline{\hat{P}_{\mathrm{f}}}$ & c.o.v. \\
\hline & $1 \times 10^{7}$ & 206.9 & $2.580 \times 10^{-5}$ & - & $1 \times 10^{5}$ & 255.0 & $1.670 \times 10^{-3}$ & - \\
\hline $\mathrm{SuS}+\mathrm{ACS}$ & $5 \times 10^{3}$ & 202.0 & $2.523 \times 10^{-5}$ & 0.333 & $3 \times 10^{3}$ & 254.3 & $1.609 \times 10^{-3}$ & 0.203 \\
\hline SuS+MMA & $5 \times 10^{3}$ & 201.8 & $2.565 \times 10^{-5}$ & 0.398 & $3 \times 10^{3}$ & 254.3 & $1.664 \times 10^{-3}$ & 0.245 \\
\hline
\end{tabular}

Alvarez, D. A., 2009b. Reduction of uncertainty using sensitivity analysis methods for infinite random sets of indexable type. International Journal of Approximate Reasoning 50 (5), 750-762.

Alvarez, D. A., Hurtado, J. E., 2014. An efficient method for the estimation of structural reliability intervals with random sets, dependence modeling and uncertain inputs. Computers \& Structures 142, 54-63.

Alvarez, D. A., Hurtado, J. E., Ramírez, J., 2017. Tighter bounds on the probability of failure than those provided by random set theory. Computers \& Structures 189, 101-113.

Alvarez, D. A., Hurtado, J. E., Uribe, F., 2014. Estimation of the lower and upper probabilities of failure using random sets and subset simulation. In: Proceedings of the Second International Conference on Vulnerability and Risk Analysis and Management (ICVRAM) and the Sixth International Symposium on Uncertainty Modeling and Analysis (ISUMA). ASCE, pp. 905-914.

Au, S.-K., Beck, J. L., 2001. Estimation of small failure probabilities in high dimensions by subset simulation. Probabilistic Engineering Mechanics 16 (4), $263-277$.

Au, S.-K., Beck, J. L., 2003. Subset simulation and its application to seismic risk based on dynamic analysis. Journal of Engineering Mechanics 129 (8), $901-917$.

Au, S.-K., Beck, J. L., Zuev, K. M., Katafygiotis, L. S., 2012. Discussion of paper by F. Miao and M. Ghosn, "modified subset simulation for reliability analysis of structural systems". Structural Safety 34 (1), $379-380$.

Au, S.-K., Patelli, E., 2016. Rare event simulation in finite-infinite dimensional space. Structural Safety 148, 67-77.

Beer, M., Ferson, S., Kreinovich, V., 2013. Imprecise probabilities in engineering analyses. Mechanical Systems and Signal Processing 37 (1), 4-29.

Bourinet, J., Deheeger, F., Lemaire, M., 2013. Assessing small failure probabilities by combined subset simulation and support vector machines. Structural Safety 33 (6), $251-260$.

Cotter, S., Roberts, G., Stuart, A., White, A., 2013. MCMC methods for functions: modifying old algorithms to make them faster. Statistical Science 28 (3), 424-446.

Couso, I., Dubois, D., Sánchez, L., 2014. Random sets and random fuzzy sets as ill-perceived random variables: an introduction for Ph.D. students and practitioners. Springer.
Crespo, L. G., Kenny, S. P., Giesy, D. P., 2013. The NASA Langley multidisciplinary uncertainty quantification challenge. 16th AIAA Non-Deterministic Approaches Conference, AIAA SciTech Forum, (AIAA 2014-1347).

Dempster, A. P., 1967. Upper and lower probabilities induced by a multivalued mapping. Annals of Mathematical Statistics 38, 325-339.

Ditlevsen, O., Madsen, H. O., 1996. Structural reliability methods. John Wiley $\&$ Sons, Inc.

Du, X., 2008. Unified uncertainty analysis by the first order reliability method. Journal of Mechanical Design 130 (9), 091401-091410.

Dubois, D., Prade, H., 1988. Possibility theory: an approach to computerized processing of uncertainty. Springer.

Dubois, D., Prade, H., 1991. Random sets and fuzzy interval analysis. Fuzzy Sets and Systems 42 (1), 87-101.

Echard, B., Gayton, N., Lemaire, M., 2011. AK-MCS: An active learning reliability method combining kriging and Monte Carlo simulation. Structural Safety 33 (2), 145-154.

Elishakoff, I., 1999. Are probabilistic and anti-optimization approaches compatible? In: Whys and Hows in Uncertainty Modelling. Springer, pp. 263-355.

Embrechts, P., Lindskog, F., McNeil, A., 2003. Modelling dependence with copulas and applications to risk management. In: Handbook of Heavy Tailed Distributions in Finance. Vol. 1: Handbooks in Finance. Elsevier/NorthHolland, Ch. 8, pp. 329-384.

Ferson, S., Kreinovich, V., Ginzburg, L., Myers, D., Sentz, K., 2003. Constructing probability boxes and Dempster-Shafer structures. Report SAND20024015, Sandia National Laboratories.

Ferson, S., Nelsen, R. B., Hajagos, J., Berleant, D. J., Zhang, J., Tucker, W. T., Ginzburg, L. R., Oberkampf, W. L., 2004. Dependence in probabilistic modeling, Dempster-Shafer theory, and probability bounds analysis. Tech. Rep. SAND2004-3072, Sandia National Laboratories.

Haario, H., Saksman, E., Tamminen, J., 2001. An adaptive Metropolis algorithm. Bernoulli 7 (2), 223-242.

Hurtado, J. E., Alvarez, D. A., 2001. Neural-network-based reliability analysis: a comparative study. Computer Methods in Applied Mechanics and Engineering 191 (1-2), $113-132$.

Hurtado, J. E., Alvarez, D. A., Barbat, A. H., 2000. Monte Carlo analysis of structural systems using neural networks. In: Proceedings of the Interna- 
tional Conference on Monte Carlo simulation (MCS-2000). Taylor \& Francis Group, pp. 265-271.

Hurtado, J. E., Alvarez, D. A., Ramírez, J., 2012. Fuzzy structural analysis based on fundamental reliability concepts. Computers \& Structures 112$113,183-192$.

Lemaire, M., Chateauneuf, A., Mitteau, J.-C., 2009. Structural reliability. Wiley-ISTE.

Molchanov, I., 2005. Theory of random sets. Springer.

Nelsen, R. B., 2007. An introduction to copulas, 2nd Edition. Springer.

Nguyen, H. T., 2006. An introduction to random sets. Chapman and Hall.

Oberguggenberger, M., Fellin, W., 2005. The fuzziness and sensitivity of failure probabilities. In: Analyzing Uncertainty in Civil Engineering. Springer, pp. 33-49.

Oberkampf, W. L., Helton, J. C., Joslyn, C. A., Wojtkiewicz, S. F., Ferson, S., 2004. Challenge problems: uncertainty in system response given uncertain parameters. Reliability Engineering and System Safety 85 (1), 11 - 19.

Papadopoulos, V., Giovanis, D. G., Lagaros, N. D., Papadrakakis, M., 2013 Accelerated subset simulation with neural networks for reliability analysis. Computer Methods in Applied Mechanics and Engineering 223-224, 70 80 .

Papaioannou, I., Betz, W., Zwirglmaier, K., Straub, D., 2015. MCMC algorithms for subset simulation. Probabilistic Engineering Mechanics 41, 89103.

Patelli, E., Alvarez, D. A., Broggi, M., Angelis, M. d., 2014. Uncertainty management in multidisciplinary design of critical safety systems. Journal of Aerospace Information Systems 12 (1), 140-169.

Potra, F. A., Wright, S. J., 2000. Interior-point methods. Journal of Computational and Applied Mathematics 124 (1-2), 281 - 302.

Rosenblatt, M., 1952. Remarks on a multivariate transformation. The Annals of Mathematical Statistics 23 (3), 470-472.

Schmitz, V., 2003. Copulas and stochastic processes. Ph.D. thesis, Fakultät für Mathematik, Informatik und Naturwissenschaften. RheinischWestfälische Technische Hochschule (RWTH) Aachen, Aachen, Germany, available at http://publications.rwth-aachen.de/record/ 59254/files/04_198.pdf.

Sklar, A., 1959. Fonctions de répartition à $n$ dimensions et leurs marges. Publications de l'Institut Statistique de l'Université de Paris 8, 229-231.

Tonon, F., 2004. Using random set theory to propagate epistemic uncertainty through a mechanical system. Reliability Engineering and System Safety 85, 169-181.

Vanmarcke, E., 2010. Random fields: analysis and synthesis. World Scientific Publishing Company.

Walter, C., 2015. Moving particles: a parallel optimal multilevel splitting method with application in quantiles estimation and meta-model based algorithms. Structural Safety 55, 10-25.

Xiao, N.-C., Li, Y.-F., Yu, L., Wang, Z., Huang, H.-Z., 2014. Saddlepoint approximation-based reliability analysis method for structural systems with parameter uncertainties. Proceedings of the Institution of Mechanical Engineers, Part O: Journal of Risk and Reliability 228 (5), 529-540.

Yang, X., Liu, Y., Gao, Y., 2016. Unified reliability analysis by active learning kriging model combining with random-set based monte carlo simulation method. International Journal for Numerical Methods in Engineering 108 (11), 1343-1361.

Zhang, H., 2012. Interval importance sampling method for finite element-based structural reliability assessment under parameter uncertainties. Structural Safety $38,1-10$.

Zhang, H., Dai, H., Beer, M., Wang, W., 2013. Structural reliability analysis on the basis of small samples: An interval quasi-Monte Carlo method. Mechanical Systems and Signal Processing 37 (1-2), 137-151.

Zhang, H., Ha, L., Li, Q., Beer, M., 2017. Imprecise probability analysis of steel structures subject to atmospheric corrosion. Structural Safety 67, $62-$ 69.

Zhang, H., Mullen, R. L., Muhanna, R. L., 2010. Interval Monte Carlo methods for structural reliability. Structural Safety 32, 183-190.

Zuev, K. M., Beck, J. L., Au, S.-K., Katafygiotis, L. S., 2012. Bayesian postprocessor and other enhancements of subset simulation for estimating failure probabilities in high dimensions. Computers \& Structures 92-93, 283 - 293.

Zuev, K. M., Katafygiotis, L. S., 2011. Modified Metropolis-Hastings algorithm with delayed rejection. Probabilistic Engineering Mechanics 26 (3), 405 412. 